\title{
Review
}

\section{Nanomaterials for analysis and monitoring of emerging chemical pollutants}

\author{
Qian Liu, Qunfang Zhou, Guibin Jiang* \\ State Key Laboratory of Environmental Chemistry and Ecotoxicology, Research Center for Eco-Environmental Sciences, Chinese Academy of Sciences, Beijing 100085, China
}

\section{A R T I C L E I N F O}

\section{Keywords:}

Carbon nanotube

Colorimetric detection

Electrochemical sensing

Emerging chemical pollutant

Environmental analysis

Gold nanoparticle

Graphene

Magnetic nanoparticle

Nanomaterial

Sample preparation

\begin{abstract}
A B S T R A C T
Emerging chemical pollutants (ECPs) are defined as new chemicals that have no regulatory status but may have an adverse impact on the environment and human health. Research on ECPs calls for new analytical tools. In this article, we review the applications of nanomaterials in analysis and monitoring of ECPs. We cover different types of nanomaterial (e.g., carbon nanotubes, graphene, metal nanoparticles, magnetic nanoparticles, and quantum dots) and different analytical techniques (e.g., sample-preparation techniques, electrochemical sensing, fluorescent detection, colorimetric detection, surface-enhanced Raman scattering, and mass spectrometry). We also discuss current challenges and give our perspectives on the future in this rapidly developing area.
\end{abstract}

(c) 2014 Elsevier Ltd. All rights reserved.

\section{Contents}

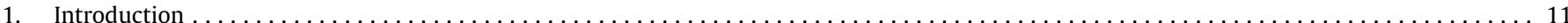

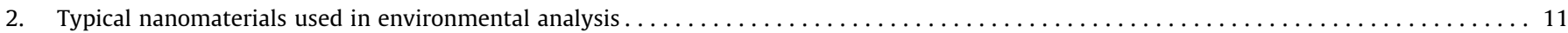

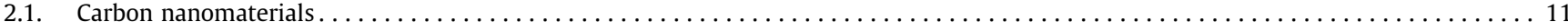

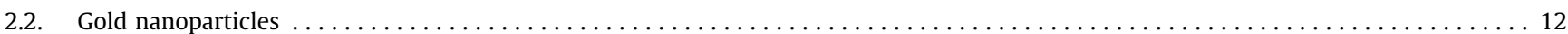

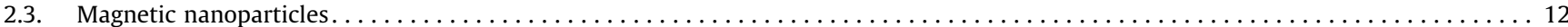

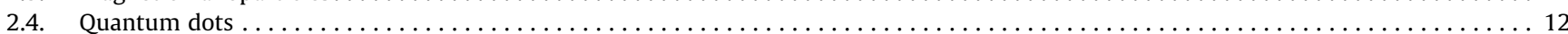

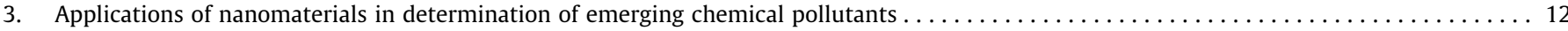

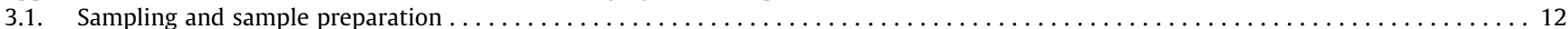

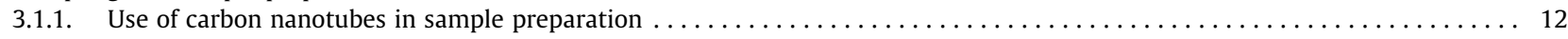

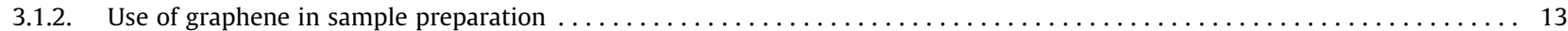

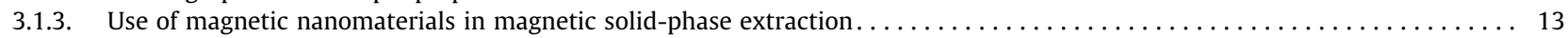

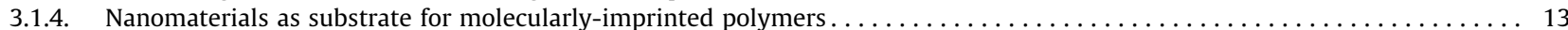

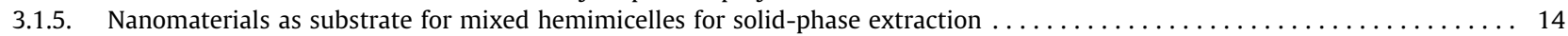

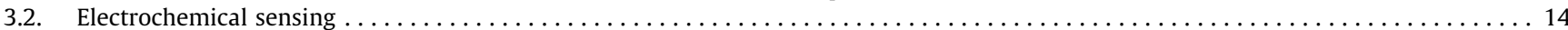

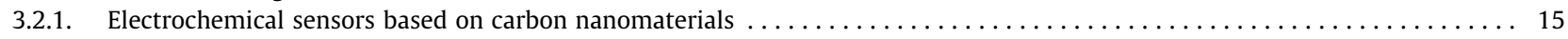

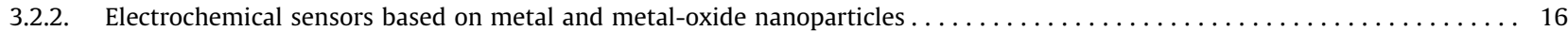

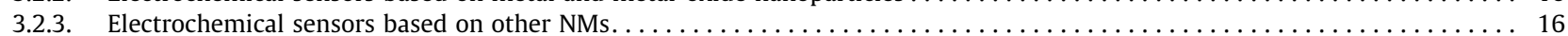

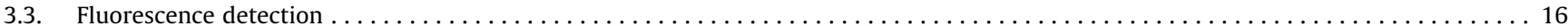

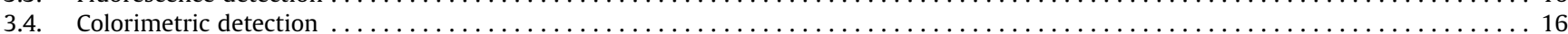

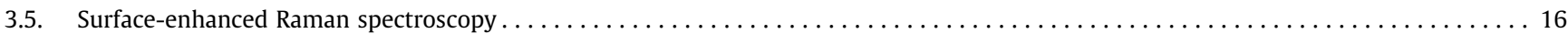

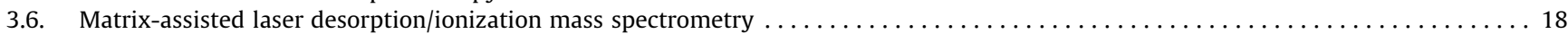

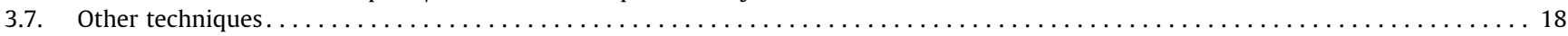

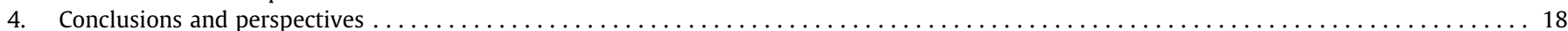

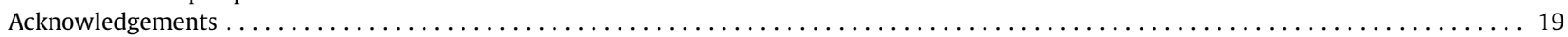

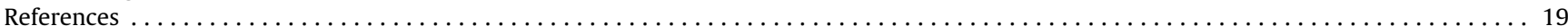

\footnotetext{
* Corresponding author. Fax: +86 1062849179

E-mail address: gbjiang@rcees.ac.cn (G. Jiang).
} 


\section{Introduction}

Tremendous efforts have been made in prevention, control, and mitigation of environmental pollution caused by persistent organic pollutants (POPs) that are known to persist in the environment, resist biodegradation, bioaccumulate in organisms, and have adverse effects on the environment and human health. Nevertheless, it was recently recognized that risks to the environment and human health are not limited to classical POPs. More and more attention is being paid to emerging chemical pollutants (ECPs), which are defined as chemicals that are not covered by standard monitoring and regulatory programs but may pose potential threatens to the environment and human health [1-3].

The open list of ECPs encompasses a wide spectrum of chemicals (Table 1). Chemicals, such as polybrominated diphenyl ethers (PBDEs) and antibiotics, have been present in the environment for decades, but only recently did they emerge into the spotlight due to advances in monitoring techniques and increased knowledge of their toxicity. Some chemicals (e.g., ionic liquids) were even thought to be safe and "green", but are now considered potential pollutants with revelation of their ecotoxicity and health effects [4]. Despite that, recognition of the occurrence of ECPs in the environment is still limited, and reliable ecotoxicological data are only available for a small fraction of these chemicals.

To understand the potential biological impact of ECPs, it is urgent to develop reliable, practical methods for identifying and quantifying these compounds in the environment. However, analysis of ECPs is a great challenge due to:

(1) the complexity of the environmental matrices;

(2) the extremely low concentrations of these chemicals in the environment (usually at parts per trillion to billion level);

(3) the presence of multiple isomers or analogs (e.g., PBDEs have 209 congeners; and short-chained chlorinated paraffins (SCCPs), with thousands of isomers, enantiomers, and diastereomers, may be one of the most complex group of organic pollutants); and,

(4) the "emerging" nature, because, some ECPs have just been identified in the environment for a short period of time, and they still lack corresponding analytical methods.

Nanomaterials (NMs) have sparked intense research interest in almost all branches of analytical chemistry due to their intriguing chemical and physical properties (e.g., size, conductivity, mechanical strength, magnetism, and light absorption and emission). The application of NMs in environmental analysis has undergone rapid growth in recent years. Specifically, NMs have shown great promise for analysis of ECPs. Many papers have reported the use of NMs in analytical method development for ECPs. These works provide new analytical tools and push forward the research on ECPs. In electrochemical sensing, NMs can be used as molecular wires to enhance electron transfer or as catalysts to accelerate chemical reactions. In optical detection, NMs can serve as optical probes for designing novel detection systems. In separation science, NMs can often be used as stationary phases or adsorbents due to their large surface area and specific affinity for the pollutant molecules. Apart from that, the use of magnetic NMs can facilitate solid-phase extraction (SPE) and save time in sample preparation, which is usually the most laborious, tedious step in environmental analysis.

In this article, we review the applications of NMs in analysis and monitoring of ECPs. We focus on emerging pollutants, and do not cover classical pollutants. Due to the large number of publications on this topic, it is impossible for us to list exhaustively all the related papers, so we select only some of the most representative papers. Incidentally, it is necessary to clarify that NMs themselves are regarded as a class of ECPs. Although present in laboratories since the early 1980s, NMs became an environmental issue only recently with their increasing use in consumer products and continual release into the environment. Some papers have reviewed safety concerns about NMs [5-8].

\section{Typical nanomaterials used in environmental analysis}

Prior to discussing the applications, it is necessary to give a brief review on the most commonly used NMs in environmental analysis, including carbon NMs, gold nanoparticles (AuNPs), magnetic nanoparticles, and quantum dots (QDs).

\subsection{Carbon nanomaterials}

Carbon NMs comprise a number of allotropes. Among them, carbon nanotubes (CNTs) and graphene have been widely used in analysis of ECPs due to their large surface area, excellent electrical and optical properties, good chemical/physical stability, and relatively low cost.

CNTs can be synthesized in large quantities by various means, such as arc discharge, high-pressure carbon-monoxide disproportionation, or chemical-vapor deposition (CVD). Some of them are also applicable for graphene (e.g., CVD and arc discharge). Moreover, graphene can be produced from graphite by chemical methods [9], namely, exfoliation of graphite to graphene oxide (GO) by harsh oxidation and sonication, followed by reduction of the GO to the parent graphene state. Notably, GO is highly soluble in water and possesses a large number of reactive groups (e.g., carboxyl and hydroxyl), so it provides a versatile precursor for functional graphene and graphene composites.

There are some issues that should be paid attention to when applying carbon NMs, as below.

(1) Metallic impurities. CNTs contain a considerable amount of residual metallic impurities from the metal catalysts used in synthesis [10]. The same situation happens to graphene if metal catalysts are used in the synthesis; otherwise, the metallic impurities may also come from natural graphite (e.g., $\mathrm{Fe}, \mathrm{Co}$ and $\mathrm{Ni}$ ) [11]. These impurities are extremely difficult to remove and can be problematic for some

Table 1

Typical emerging chemical pollutants mentioned in this review

Classification

Pharmaceuticals and personal-care products (PPCPs)

Flame retardants

Perfluorinated compounds (PFCs)

Industrial additives

Others
Typical compounds

Antibiotics; Steroids and hormones; Non-steroidal anti-inflammatory drugs (NSAIDs); Anticonvulsant and antidepressant drugs; $\beta$-Blockers; Lipid regulators; Triclosan; UV filters

Polybrominated diphenyl ethers (PBDEs); Tetrabromobisphenol A (TBBPA); Tris(2,3-dibromopropyl) isocyanurate (TBC) Perfluorooctanoic acid (PFOA); Perfluorooctanesulfonic acid (PFOS)

Bisphenol A (BPA); Alkylphenols; Phthalate esters; Methyl tert-butyl ether (MTBE); Short-chained chlorinated paraffins (SCCPs)

Disinfection by-products (DBPs); Perchlorate; Nanomaterials (NMs); Algal toxins

\footnotetext{
${ }^{\text {a }}$ In this review, only papers that treated PPCPs as pollutants are discussed, so some medical-oriented applications are not involved.
} 
applications, so it is necessary to exclude or to discriminate the effects of metallic impurities from the material itself in the application.

(2) Heterogeneity. CNTs differ significantly in length, diameter, and chirality (determining CNTs to be semiconducting or metallic); and, graphene also contains large variations in lateral size, shape, and thickness. From the viewpoint of analytical chemistry, these variations may bring in more uncertainties to analytical results, so it is highly desirable to obtain carbon NMs with uniform morphology via controlled synthesis or post-synthesis purification.

(3) Agglomeration. Carbon NMs have a strong tendency to agglomerate via Van der Waals forces or $\pi-\pi$ stacking. This may seriously compromise the exceptional properties associated with only individual CNTs or nanosheets. Using stabilizers (i.e., surfactants) or modifying the pristine carbon NMs (i.e., acidification) can effectively mitigate the agglomeration.

(4) Irreversible adsorption. In adsorption-related applications, irreversible adsorption of the target analytes on carbon NMs should be prevented, as this may cause low recovery and detract from the reusability of the materials.

\subsection{Gold nanoparticles}

AuNPs are the most popular metal NPs in environmental analysis. One of the reasons is that AuNPs are very easy to prepare in a liquid by reducing chloroauric acid with a reductant. For example, nearly monodisperse spherical AuNPs with 10-20 nm diameter can be prepared using sodium citrate as both reducing agent and capping agent. Occasionally, silver NPs (AgNPs) were used as alternatives to AuNPs in environmental analysis.

Rational design of the coatings on AuNPs is usually the key point for application. For example, AuNPs can be used as a visual probe in colorimetric detection based on the intrinsic plasmon-resonance absorption, which is sensitive to the distance between particles. To achieve the goal, the AuNPs need to be covalently or noncovalently modified with a responsive coating, which allows the target analyte to trigger the aggregation of AuNPs by replacement or conformation change of the coating molecules. For analysis of ECPs, the coatings involve nucleic acids (e.g., aptamers), antibodies, polymers [e.g., molecularly-imprinted polymers (MIPs)], or small molecules that have a specific interaction with the analyte. As another example, in surface-enhanced Raman scattering (SERS), AuNPs can dramatically enhance the Raman scattering signals when the molecules are close to the AuNP surface that has a very high curvature. Again the surface of AuNPs needs to be modified with a coating that can specifically bind the analyte.

\subsection{Magnetic nanoparticles}

Magnetic NPs (MNPs), especially ferromagnetic NPs, plays a unique role in modern SPE and other analytical techniques. MNPs can not only provide high adsorption capacity, but also endow the adsorbent with responsiveness to external magnetic fields. This feature allows rapid separation from the bulk solution and easy reuse of the adsorbent without centrifugation, filtration or packing into a cartridge, thus greatly facilitating the sample-preparation process. Besides, MNPs are often used as a support for other materials or integrated into nanocomposites to introduce magnetism to the materials.

Co-precipitation is the easiest way to synthesize $\mathrm{Fe}_{3} \mathrm{O}_{4}$ MNPs by adding a base to aqueous $\mathrm{Fe}^{2+} / \mathrm{Fe}^{3+}$ salt solutions under inert atmosphere and vigorous stirring. Deliberate oxidation of the prepared $\mathrm{Fe}_{3} \mathrm{O}_{4} \mathrm{NPs}$ can convert them to more stable $\gamma-\mathrm{Fe}_{2} \mathrm{O}_{3} \mathrm{NPs}$. Other methods for synthesizing MNPs include thermal decomposition, microemulsion, and hydrothermal synthesis [12].
In applying MNPs, a knotty problem is the stability of the NPs because they are easily oxidized to maghemite under ambient conditions. MNPs are also prone to agglomerate or to precipitate in aquatic solutions, so MNPs are usually protected by a coating (e.g., surfactant, polymer, metal, carbon or silica) to prevent oxidation by oxygen. In most cases, the protecting coating also works as a stabilizer. Among these coatings, silica coating is especially interesting because it is easy to prepare (e.g., using the Stöber method or the sol-gel process) and is favorable for further functionalization.

\subsection{Quantum dots}

QDs have unique size-dependent fluorescence, high fluorescence quantum yields, narrow spectral line widths, and stability against photobleaching. These properties enable use of QDs as reliable optical labels in environmental analysis. Also, QDs are photoelectrochemically active, so they have potential to be applied in (photo)electrochemical sensors [13].

Besides the above-mentioned NMs, there are other NMs that have gained some applications in analysis of ECPs, such as silica NPs $\left(\mathrm{SiO}_{2} \mathrm{NPs}\right), \mathrm{TiO}_{2} \mathrm{NPs}$ or nanotubes, and polymeric NPs.

\section{Applications of nanomaterials in determination of emerging chemical pollutants}

\subsection{Sampling and sample preparation}

Application of NMs in sample preparation is a very active research area. Many efforts have been made to establish preconcentration methods using NMs as adsorbents. Since ECPs comprise a wide range of chemicals differing greatly in properties (e.g., polarity, water solubility, and volatility), the selection of NMs and extraction techniques highly depends on the nature of the analytes. The current trends in this aspect mainly include the following.

\subsubsection{Use of carbon nanotubes in sample preparation}

Since the first report by Cai et al. [14] that used multi-walled CNTs (MWCNTs) as SPE adsorbent for determination of bisphenol A (BPA) and alkylphenols in waters, the application of CNTs in sample preparation has been studied intensively. The simplest mode is packing CNTs directly into a commercial cartridge for SPE [14,15]. Dahane et al. [16] recently used an MWCNT-packed cartridge to extract four $\beta$-blockers and eight non-steroidal anti-inflammatory drugs (NSAIDs) in river water and wastewater. They found that using MWCNTs can reduce the matrix effect, and the cost was lower than that of commercially-available cartridges. Also, CNTs can also be integrated into on-line SPE systems coupled to HPLC [17] or CE-MS [18] for ECP analysis, and used in dispersive SPE to improve the analytical throughput [19-21].

Besides SPE, CNTs have also gained much interest in solid-phase microextraction (SPME). Since CNTs are difficult to dissolve or to disperse in common solvents or polymeric matrices, it is critical to find a proper way to immobilize CNTs on the SPME fiber. The available methods include physical adsorption [22], electrophoretic deposition [23], and sol-gel processes [24-28]. Zhang et al. [24] modified the acid-oxidized single-walled CNTs (SWCNTs) with hydroxyl-terminated silicone oil to improve the solubility of SWCNTs in organic solvents, and then prepared a SWCNTenhanced polymeric coating via a sol-gel process to create a porous structure on the surface of the fiber. This fiber was used to extract PBDEs in water samples in headspace mode with higher extraction efficiency, better thermal stability and longer life span than commercial fibers. In other reports [26,28], MWCNTs were grafted with poly(ethylene glycol) (PEG) and used as a stationary phase to 
prepare sol-gel SPME fibers. The fiber was applied in extraction of NSAIDs [26] and methyl tert-butyl ether (MTBE) [28] in water samples.

CNTs have been used to enhance the performance of hollow fiber-liquid-phase microextraction (HF-LPME) [29-31]. Song et al. [29] incorporated the MWCNTs into the pores of the HF via a solgel method for the analysis of piroxicam and diclofenac in water samples. The analytes were extracted by both the organic solvent inside the HF and the MWCNTs, thus yielding higher extraction efficiency than normal methods.

\subsubsection{Use of graphene in sample preparation}

Graphene has shown potential to replace CNTs in sample preparation, mainly due to its unique two-dimensional structure and more friendly methods of synthesis. A more specialized review paper on this topic was published in this journal [32], so, herein, we focus only on new progress related to ECPs.

For SPE application, like CNTs, graphene can be directly packed into a SPE cartridge [33]; however, there still are concerns about agglomeration of graphene and loss of miniscule graphene sheets. Thus, researchers have also tried to combine graphene with other materials to avoid potential problems and still maintain its advantageous properties. For example, Liu et al. [34] synthesized graphene- and GO-bound silica and used them as adsorbents for reversed-phase and normal-phase SPE, respectively. The former was applied to extraction of chlorophenols in water and the latter to hydroxylated PBDEs in organic solvent. In both modes, better or comparable performance was achieved than with commerciallyavailable materials. Yu et al. [35] used a hydrothermally-treated graphene/silica gel as an SPE adsorbent for analysis of nine PPCPs in wastewater. The graphene showed better extraction efficiency than Oasis HLB adsorbent.

For SPME application, Zhang et al. [36] developed a plunger-inneedle microsyringe using the plunger wire as coating support and graphene as sorbent for determination of PBDEs in canal-water samples. The graphene coating was prepared via a sol-gel approach to create a porous structure and enhance the thermal stability of the coating. Compared with commercial fibers, higher extraction efficiency and selectivity for PBDEs were achieved.

Recently, Liu et al. [37] reported the fabrication of a graphenized fiber by in-situ chemical exfoliation of graphite in pencil lead to graphene sheets via a simple pressurized oxidation reaction for extraction of four bisphenol analogs [BPA, bisphenol S, bisphenol $\mathrm{AF}$, and tetrabromobisphenol A (TBBPA)] from water samples. This fiber combined the low cost of pencil lead with the excellent extraction capability of graphene, and also displayed a long lifespan and good tolerance to harsh usage conditions.

Liu et al. [38] reported the graphene-assisted matrix-assisted solid-phase dispersion (MSPD) method for extraction of PBDEs and their methoxylated and hydroxylated analogs from solid environmental samples (i.e., soil, tree bark, and fish). They found that graphene allowed sufficient dispersion of the sample matrix. Better recoveries were obtained with graphene than with other sorbents (e.g., C18, Florisil and CNTs) and other extraction techniques (e.g., Soxhlet and accelerated solvent extraction).

Due to its unique planar structure, graphene also provides an ideal substrate for other adsorbent materials (e.g., MIPs, magnetic NMs, and mixed hemimicelles), which we describe in the following sections.

\subsubsection{Use of magnetic nanomaterials in magnetic solid-phase extraction}

Magnetic SPE developed rapidly in recent years due to its clear advantages, such as easy operation, high extraction efficiency, and reusability of adsorbent. Since bare MNPs are easily oxidized to lose their magnetism or agglomerate in solutions, they are usually used after

(1) protection by a functional layer or a coating to form a coreshelled nanostructure; and,

(2) hybridization with other materials to introduce magnetism to the composite adsorbent.

In core-shelled structures of MNPs, different functional groups have been employed as the outer layer to offer good selectivity for the analytes, such as polymers [39,40], metal oxide [41], carbon [42], organic groups [43-46], surfactants [47-49], and $\beta$-cyclodex$\operatorname{trin}(\beta-C D)[50]$.

Zhang et al. [44] synthesized chitosan/octadecyl-functionalized $\mathrm{Fe}_{3} \mathrm{O}_{4} \mathrm{NPs}$ and used it as an adsorbent to extract PFCs from wastewater. The $\mathrm{Fe}_{3} \mathrm{O}_{4} \mathrm{NP}$ core allowed rapid magnetic separation of the adsorbent from the bulk solution and reuse of the adsorbent, the $\mathrm{C}_{18}$ interior layer could trap the analytes, and the chitosan outer layer improved the dispersibility of the adsorbent in aqueous solutions and suppressed the interferences from natural organic substances.

Román et al. [43] synthesized chemisorbed oleic acid-coated cobalt ferrite ( $\mathrm{CoFe}_{2} \mathrm{O}_{4} @$ oleic acid) MNPs to preconcentrate UV filters in water samples of different origins (tap, river and sea). The cobalt-ferrite NPs were reported to have better chemical stability against oxidation than magnetite-based NPs.

Niu et al. [42] prepared core-shell structured carbon-coated MNPs by using glucose as a carbon source (Fig. 1). This material has been used to extract different types of ECPs (e.g., BPA, alkylphenols, phthalates, and antibiotics) in water samples. The oxygen-containing species on the carbon shell of the carboncoated MNPs can be controlled by heating the material at different temperatures, and, in this way, the adsorption of the analytes on the NPs can be tailored to achieve the best results.

The hybridization of magnetic-carbon NMs is interesting because it combines the strong extraction capability of carbon NMs with the operation convenience of magnetic NMs [51-55]. Luo et al. [56] prepared MWCNT- $\mathrm{Fe}_{3} \mathrm{O}_{4}$ nanocomposites by a simple adsorption method and used the nanocomposites for fast extraction of phthalate-acid esters from beverage, water and perfume samples. This method was also used by the same group [57] to prepare graphene- $\mathrm{Fe}_{3} \mathrm{O}_{4} @ \mathrm{SiO}_{2}$ nanocomposites for extraction of six sulfonamide antibiotics from water samples.

\subsubsection{Nanomaterials as substrate for molecularly-imprinted polymers}

Molecular imprinting is a powerful technique to improve the selectivity of the method by using MIPs as the adsorbents. Unfortunately, this technique is so far feasible for only a limited number of pollutants. For MIPs, it is key to select proper template molecules and polymeric monomers to ensure complete template removal and efficient analyte adsorption and elution. Construction of MIPs on a nano-substrate can further improve the extraction efficiency of the method.

Different types of NMs have been used as substrates for MIPs. Gao et al. [58] synthesized core-shell MIPs on silica-coated MWCNTs via a sol-gel process for determination of triclosan in environmental water samples. The MIPs exhibited fast adsorption kinetics, high capacity, and good selectivity.

Liu et al. [59] used silica-protected $\mathrm{Fe}_{3} \mathrm{O}_{4}$ MNPs as a substrate for MIPs toward pefloxacin mesylate in egg samples. Since pefloxacin mesylate is a highly water-soluble molecule, a novel surfaceinitiated atom transfer radical polymerization reaction was used to synthesize the MIPs, which allowed the synthesis to proceed in a highly polar solvent (e.g., methanol/water). 


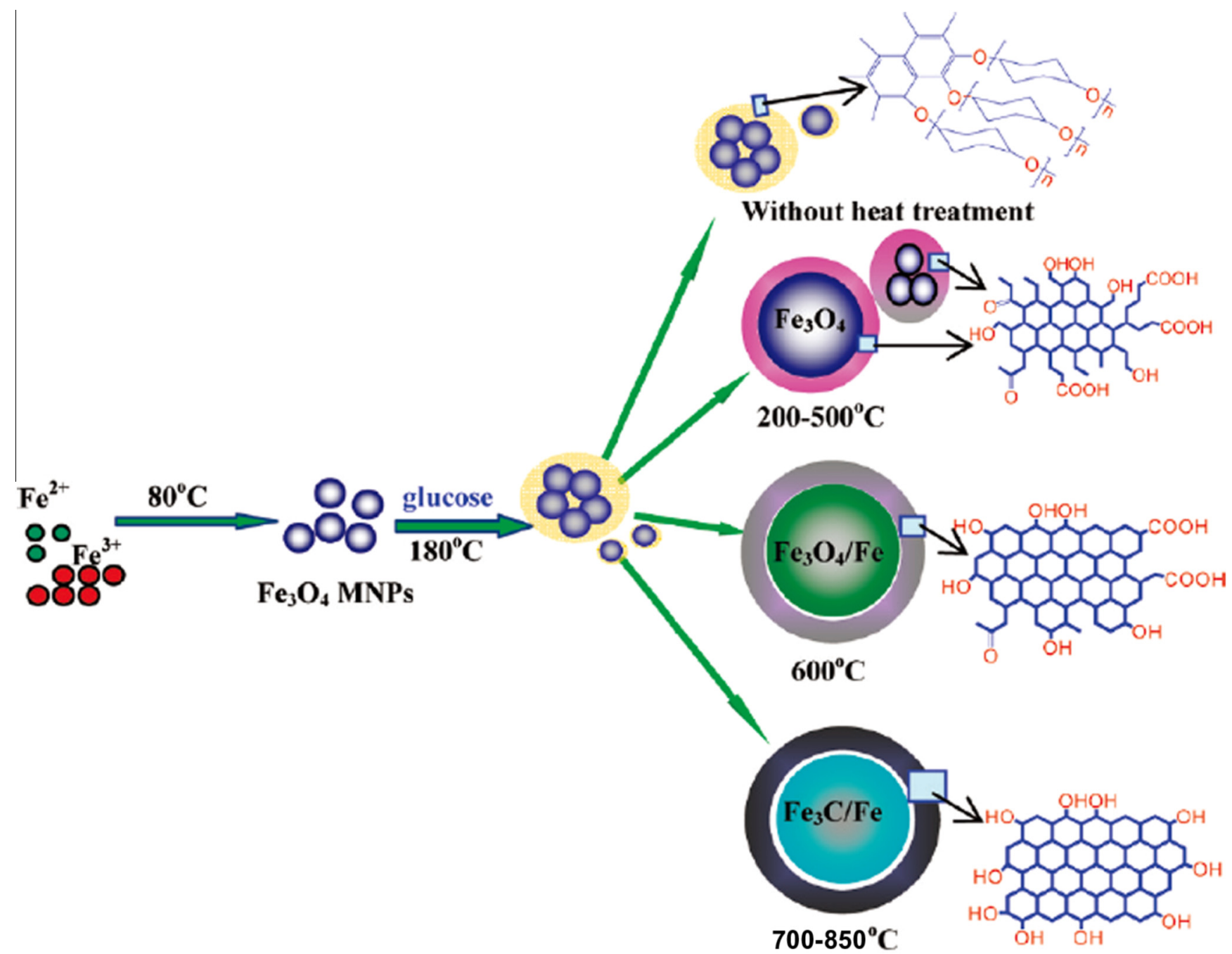

Fig. 1. Preparation of carbon-encapsulated magnetic nanoparticles with different surface properties [42] (Reprinted with permission from American Chemical Society).

Lin et al. [60] used bisphenol $\mathrm{F}$ as a dummy template to prepare the MIP of BPA with core-shell MNPs as support. The dummy MIPs could distinguish BPA, TBBPA and p-tertbutylphenol.

Wei et al. [61] prepared SiNPs by the Stöber method as the substrate for MIP. The method was applied in determination of BPA in fish samples and showed higher selectivity and better recovery than $\mathrm{C} 18$ adsorbent.

\subsubsection{Nanomaterials as substrate for mixed hemimicelles for solid- phase extraction}

The mixed hemimicelle-based extraction technique uses the hemimicelles (monolayers) and admicelles (bilayers) formed by a surfactant as adsorbents. The analytes can be strongly adsorbed via multiple mechanisms: hydrophobic or chain-chain interactions with the hydrocarbon chains of the surfactant, or electrostatic interaction or hydrogen bonding with the polar groups, so this technique is particularly suitable for amphiphilic analytes or those bearing long alkyl chains \{e.g., perfluorinated compounds (PFCs) [62], alkylphenols [62-64], and phthalate esters [65,66]\}.

Zhao et al. [63] investigated the use of mixed cetyltrimethylammonium bromide (CTAB) hemimicelle-coated $\mathrm{Fe}_{3} \mathrm{O}_{4}$ MNPs for extraction of BPA and alkylphenols from water samples. A high concentration factor of 800 was achieved. The same group [64] then replaced the bare $\mathrm{Fe}_{3} \mathrm{O}_{4} \mathrm{NPs}$ by silica-protected $\mathrm{Fe}_{3} \mathrm{O}_{4} \mathrm{NPs}$ to enhance the stability of the MNPs, and the concentration factor was further improved to 1600 .
Liu et al. [62] synthesized magnetic graphene sheets for formation of mixed hemimicelles for extraction of PFCs, alkylphenols and alkyltrimethylammonium salts from water samples. The $\mathrm{Fe}_{3} \mathrm{O}_{4} \mathrm{NP}-$ decorated graphene was synthesized by a direct redox reaction between $\mathrm{GO}$ and $\mathrm{Fe}(\mathrm{II})$ ions, and exhibited higher loading capacity for mixed hemimicelles than only $\mathrm{Fe}_{3} \mathrm{O}_{4} \mathrm{NPs}$. The extraction can be conducted in cationic or anionic mode by using different charged surfactants (CTAB or SDS).

In addition to the cases mentioned above, some other interesting NMs used in sample preparation include mesoporous cellular foams for TBBPA [67], Nylon 6 nanofibers for estrogens and phthalate esters $[68,69]$, and $\mathrm{TiO}_{2} \mathrm{NPs}$ for phthalate esters [70].

\subsection{Electrochemical sensing}

Electrochemical sensors are of great interest due to the flexibility in functional design, high sensitivity, low cost, and ease of operation. Regarding ECPs, most electrochemical sensors are developed for phenolic compounds (especially BPA), pharmaceuticals, and toxins. However, for halogenated organic compounds, only a few electrochemical methods have been reported, probably due to the low electrochemical activity of these compounds.

Integration of NMs into electrochemical sensors can often dramatically improve the analytical performance. The NMs can accelerate electron transfer, adsorb the target pollutants, enlarge the surface area of the electrode, immobilize bio-active molecules 


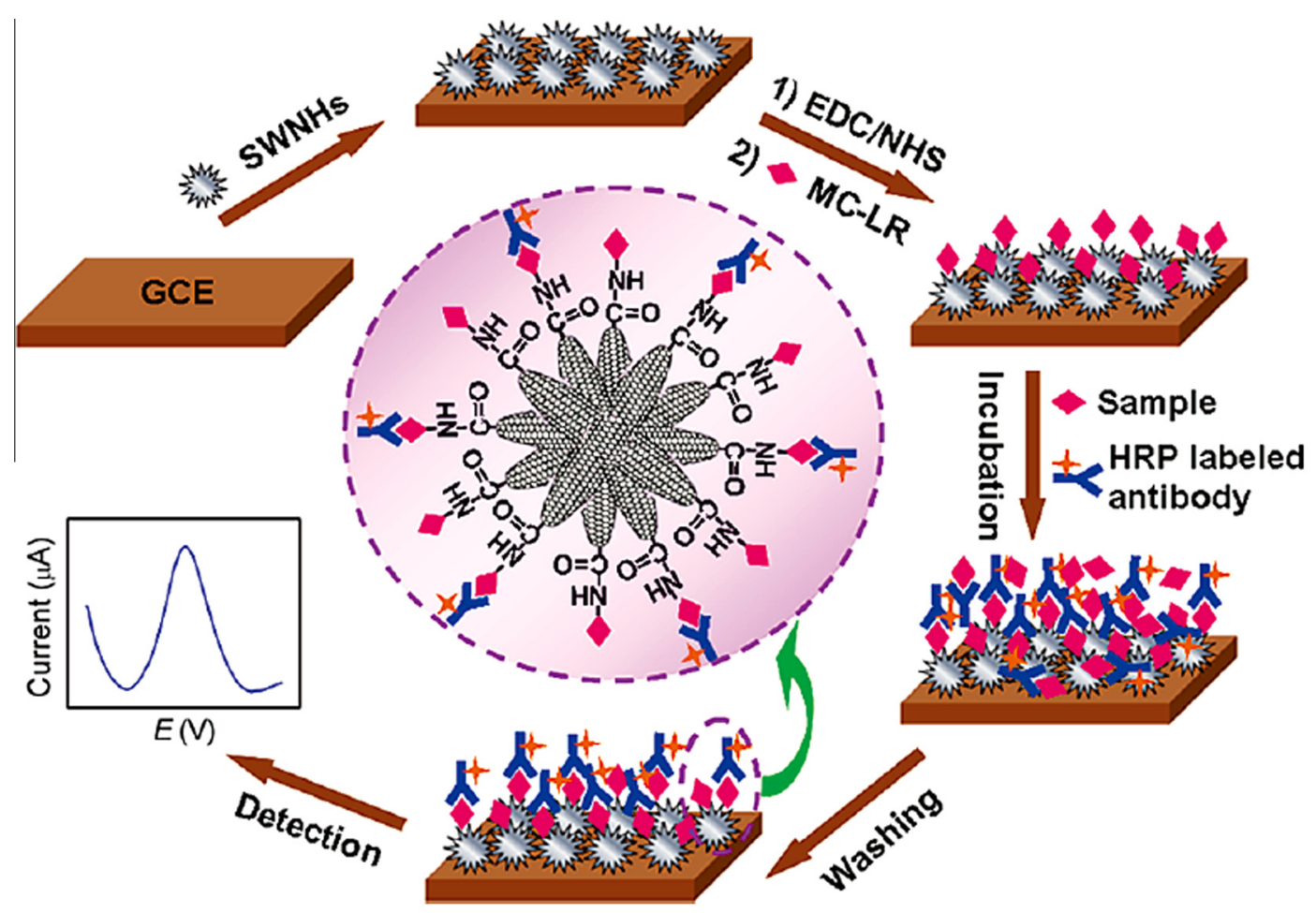

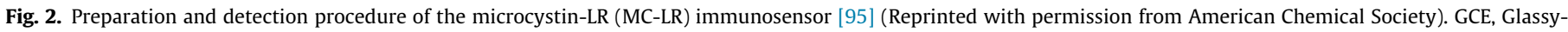

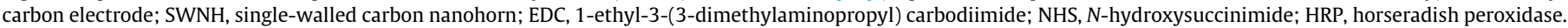

(e.g., enzyme or aptamer), and serve as electrochemical probes. In many cases, multiple types of NM were used jointly to build a multi-functional sensing interface.

\subsubsection{Electrochemical sensors based on carbon nanomaterials}

Generally, CNTs are applied in electrochemical sensing of ECPs in the following modes:

- directly modifying the electrode with CNTs to improve the response [71,72];

- hybridization of CNTs with other materials (e.g., AuNPs [73], SbNPs [74], ionic liquid [75], graphene [76], dye [77], and organometallic compound [78]) to achieve synergetic effects;

- modifying the CNTs or using them together with other recognizing units (e.g., MIP [73], antibody [75], aptamer [79], $\beta-C D$ [80], and melamine [81]) to improve the selectivity of the sensor.

Vega et al. [71] reported the use of a CNT-modified glassy-carbon electrode (GCE) for the determination of phenolic estrogens after HPLC separation. Moraes et al. [82] found that the surface organization of CNTs exerts a remarkable effect on the electrode performance. The vertically-aligned CNTs showed a stronger electrocatalytic effect than randomly-dispersed CNTs toward BPA.

Parra et al. [72] developed a solid-contact ion-selective electrode for detecting perchlorate in water based on MWCNTs as the transducer material. The selectivity was achieved by using an ion-exchange polymeric membrane containing a synthesized perchlorate salt. The limit of detection (LOD) of perchlorate was $10^{-7.4} \mathrm{M}$.

The application modes of graphene are similar to those of CNTs Graphene can be directly used to modify the electrode. For example, Wan et al. [83] investigated the electrochemical properties of graphene nanoplatelets on a GCE and used the GCE for detection of endocrine-disrupting chemicals (i.e., BPA, 2,4dichlorophenol, and octylphenol).

Furthermore, graphene has been hybridized or combined with other materials, such as AuNPs [84-86], $\mathrm{Fe}_{3} \mathrm{O}_{4} \mathrm{NPs}$ [87,88], tyrosinase $[89,90]$, chitosan $[91,92]$, ionic liquids [85,92], conducting polymer [85], MIPS [93], and mesoporous alloy [94]. Fan et al. [91] used nitrogen-doped graphene sheets along with chitosan to modify a GCE for BPA detection. N-doped graphene was reported to have a better electron-transfer ability and electrocatalytic activity than normal graphene. The LOD for BPA was $5.0 \times 10^{-9} \mathrm{M}$.

Gutés et al. [86] synthesized a decabromodiphenyl ether (BDE-209, one of PBDEs)-binding peptide and used it as a selective capturer for BDE-209. This special peptide was then assembled on AuNP-decorated graphene for detection of BDE-209 by impedance spectroscopy.

Chen et al. [93] developed an indirect electrochemical method for detection of TBBPA in waters by constructing a MIP layer on a graphene-modified carbon electrode. Because the direct electrochemical response of TBBPA is poor, $\left.\mathrm{Fe}(\mathrm{CN})_{6}^{3-}\right|^{4-}$ was used as an electro-active probe, which could form complexes with TBBPA. The LOD of TBBPA is $0.23 \mathrm{nM}$.

In addition to CNTs and graphene, a special form of carbon NMs, namely carbon nanohorns (CNHs), has also been applied in electrochemical sensing of ECPs. Zhang et al. [95] proposed an electrochemical immunosensor based on single-walled $\mathrm{CNHs}$ (SWCNHs) for detection of microcystin-LR (MC-LR) in water samples (Fig. 2). The CNHs were first modified with MC-LR and immobilized to a GCE, and then a horseradish peroxidase (HRP)-labeled MC-LR antibody was added. The MC-LR in the sample could competitively bind the HRP-labelled antibody and thus caused the electrode signal to decrease. In this assay, CNHs showed better sensitizing effect than SWCNTs, and the LOD for MC-LR was $0.03 \mu \mathrm{g} / \mathrm{L}$.

In another report, $\mathrm{Xu}$ et al. [96] used a CNH-modified electrode for BPA detection with an LOD of $1.8 \mu \mathrm{M}$. 


\subsubsection{Electrochemical sensors based on metal and metal-oxide nanoparticles}

Various metal and metal-oxide NPs with different morphologies, such as AuNPs [97-99], NiNPs [100], CdSNPs [101], $\mathrm{TiO}_{2}$ nanotubes [102], and ZnO nanotetrapods [103], have been used in electrochemical sensing of ECPs as electroactive probe or electrode modifier. In the last section, we introduced some applications that combined AuNPs with carbon NMs [73,84-86]. Besides that, AuNPs have also been modified with aptamers [98], lipid bilayers [97], and MIPs [99].

Xia et al. [97] fabricated a biosensor by immobilizing estrogen receptors in a supported bilayer lipid membrane modified with AuNPs for detection and monitoring of estrogenic chemicals in water samples. The LOD for $17 \beta$-estradiol was $1 \mathrm{ng} / \mathrm{L}$.

Alkasir et al. [100] utilized NPs as tyrosinase-immobilization matrices for biosensing of BPA. They compared the performance of magnetic NiNPs with $\mathrm{Fe}_{3} \mathrm{O}_{4}$ NPs and AuNPs, and found that the NiNPs yielded the highest sensitivity (the LOD for BPA was $7.1 \times 10^{-9} \mathrm{M}$ ).

\subsubsection{Electrochemical sensors based on other NMs}

Other NMs have also been employed in electrochemical sensing of ECPs. For example, Yin et al. [104] used CoTe QDs and a poly(amidoamine) dendrimer to modify a GCE for selective determination of BPA in water samples. The LOD was $\sim 1 \times 10^{-9} \mathrm{M}$.

Wang et al. [105] reported a BPA electrochemical sensor based on mesoporous silica MCM-41. The MCM-41 showed a better ability of signal enhancement than CNTs, activated carbon, silica gel and graphite, yielding an LOD of $3.8 \times 10^{-8} \mathrm{M}$.

Carvalho et al. [106] developed a sensor array ("electronic tongue") to detect carcinogenic trihalomethanes (THMs), a class of DBPs, in water supply. The sensor array was built by layer-by-layer depositing conducting and natural polymers on Au-interdigitated electrodes to obtain nanostructured films. The LOD for the THMs was $0.02 \mathrm{mg} / \mathrm{L}$.

\subsection{Fluorescence detection}

In fluorescence detection, NMs can play two distinct roles:

- fluorophores (e.g., QDs [107-110], GO [111], and Eu-based NPs [112]); or,

- quenchers (e.g., AuNPs [111] and graphene [113,114]).

Moreover, MNPs can be used as a loader for other fluorophores to offer convenience in operation [115-117].

Chen et al. [110] used MIP-coated Mn-doped ZnS QDs for selective detection of TBBPA in water and soils. The MIP film was prepared with diphenolic acid as a dummy template. This sensor showed good selectivity towards TBBPA and the LOD was $0.015 \mu \mathrm{M}$.

Shi et al. [111] reported a biosensor for microcystins (MCs) based on fluorescence resonance-energy transfer (FRET) between AuNPs and fluorescent GO. The GO was covalently linked with the MC antibody, and the AuNPs were modified with single-strand DNA. The MCs could bind to both the antibody and the DNA, thus inducing the fluorescent quenching of GO by AuNPs. With this sensor, the LODs were $0.5 \mathrm{mg} / \mathrm{L}$ and $0.3 \mathrm{mg} / \mathrm{L}$ for MC-LR and MC-RR, respectively.

Davydov et al. [112] synthesized fluorescent NPs by using Euorganic complexes as core and multilayered polyelectrolytes as a shell for detection of fluoroquinolone antibiotics. The fluorescence of these Eu-based NPs can be quenched by the analytes via ternary complex formation and ligand exchange.

Tan et al. [118] reported that the fluorescence of $\mathrm{Eu}(\mathrm{III})$ can be strongly enhanced by AgNPs through a metal-enhanced fluorescence effect, and applied this phenomenon in detection of tetracycline in milk with an LOD of $4 \mathrm{nM}$.

Due to its strong light-absorption ability over the whole wavelength region, graphene can be used as a highly efficient quencher in fluorescence detection. Liu et al. [114] reported a homogeneous competitive fluoroimmunoassay for detection of MC-LR in water samples. This assay utilized the quenching ability of graphene to the fluorescently-labeled DNA-MC-LR conjugates and the competitive binding with anti-MC-LR. The LOD achieved for MC-LR was $0.14 \mu \mathrm{g} / \mathrm{L}$.

Zhao et al. [113] reported the use of fluorescently-labeled aptamer-graphene conjugates as a probe for oxytetracycline detection. The analyte could detach the aptamer from the graphene and thus restore the fluorescence. The LOD was $10 \mathrm{nM}$.

\subsection{Colorimetric detection}

Visual colorimetric detection is attractive due to its low cost, simple operation, and possibility of detection with the naked eye, so it has potential to be applied in on-site monitoring of environmental pollution. AuNPs play a major role in colorimetric detection due to its high extinction coefficient. A general design is to protect the AuNPs with a functional group or molecule (e.g., DNA aptamers, or polyelectrolyte). Such a protection agent should be able to bind specifically with the anlayte and dissociate from the AuNPs, thereby allowing aggregation of AuNPs induced by salt (along with the colloid color changing from red to blue). This mechanism has been adopted in detecting BPA [119,120], oxytetracycline [121], kanamycin [122], and perfluorooctanoic acid (PFOA) [123] in water samples.

Wang et al. [124] reported an optical sensor for MC-LR based on the assembly of gold nanorods (AuNRs). The AuNRs, modified with two proteins, MC-LR antibody and MC-LR-ovalbumin antigen, were able to assemble side-by-side or end-to-end. The MC-LR in the sample can interrupt such assemblies, thus causing a shift in the UV/visible absorption and dynamic light-scattering signals. High sensitivity ( $5 \mathrm{ng} / \mathrm{L}$ ) for MC-LR was achieved with this sensor.

The same group [125] also used this method to detect gentamicin, a broad spectrum aminoglycoside antibiotic, with an LOD of $0.05 \mathrm{ng} / \mathrm{mL}$.

Another interesting application is the development of AuNPbased visual lateral flow strip sensors. Lateral flow strip devices, with the most famous examples of pregnancy testing strip and glucose testing, are particularly suitable for on-site monitoring.

Mei et al. [126] designed a lateral flow strip for visual detection of BPA based on competitive immunoadsorption (Fig. 3A). The BPA antibody-conjugated AuNPs were used as the visual probe. BPAbovine serum albumin (BSA) conjugates and goat-anti-mouse antibody were immobilized on the nitro-cellulose membrane as the test and control line, respectively. This device provides a simple way to detect BPA. However, the sensitivity is not satisfactory (LOD $5 \mu \mathrm{g} / \mathrm{L}$ ), so the same group [127] also developed a signalamplified lateral flow strip for BPA detection (Fig. 3B). Since the BPA antibody-conjugated AuNPs were blocked by BSA, a second type of AuNP modified with anti-BSA was used as an "enhancement pad". The second AuNPs could further label the first AuNPs, and thus enhanced the analytical sensitivity. The LOD for BPA is $0.5 \mu \mathrm{g} / \mathrm{L}$ with the naked eye.

\subsection{Surface-enhanced Raman spectroscopy}

SERS is a highly sensitive analytical technique and can provide structural information of the analytes in complex samples, so SERS is suitable for the detection of trace amounts of ECPs in environmental samples. The SERS signals strongly depend on the substrates used, which are normally nanostructured Au or Ag. 
(A) Negative

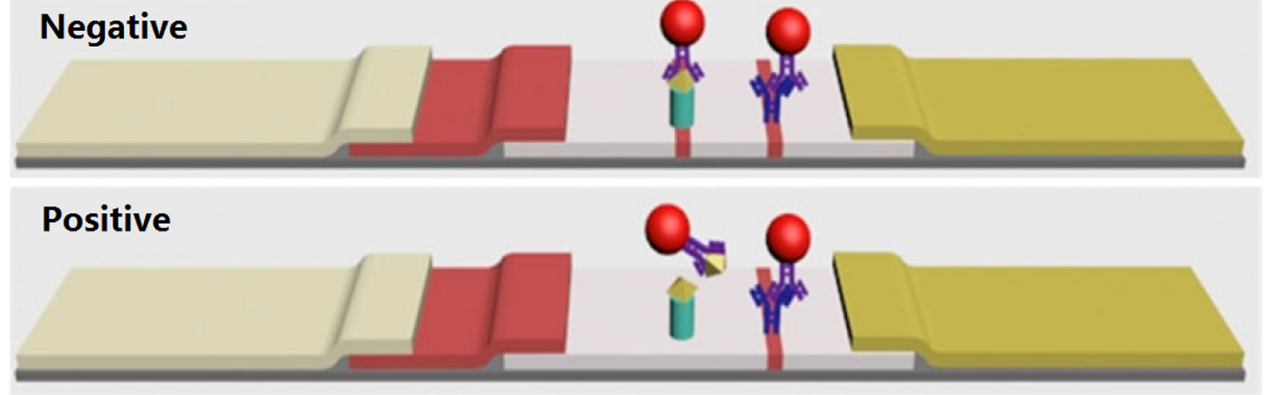

(B)

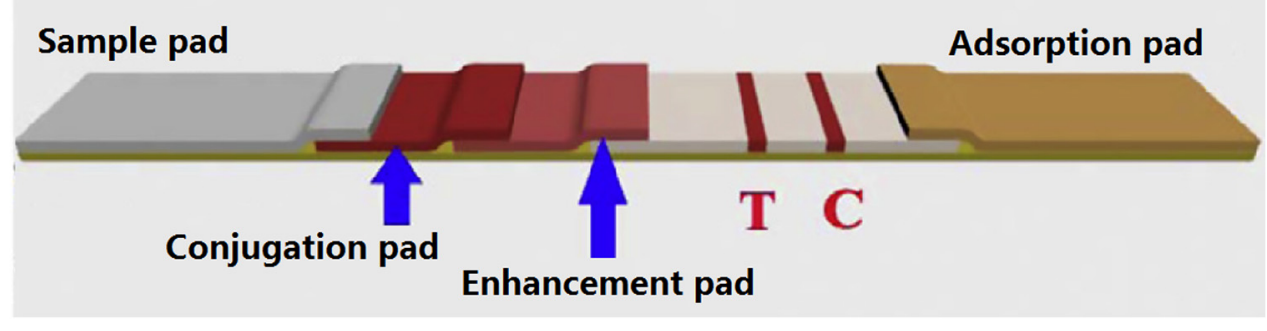

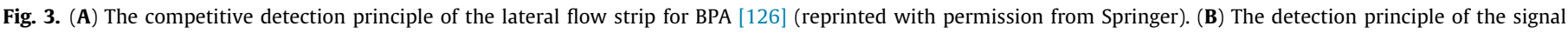
amplified lateral flow strip for BPA [127] (Reprinted with permission from Elsevier). T, Test line; C, Control line.

A series of works have been carried out on SERS detection of perchlorate in waters using different substrates, such as AuNPs [128], Au-Si nanocomposites [129], and Ag nanofilms [130,131]. The highest sensitivity for perchlorate (LOD $5 \mu \mathrm{g} / \mathrm{L}$ ) was achieved with cysteamine-modified Ag nanofilms as a SERS substrate [131].

Li et al. [132] developed a disposable Ag-graphene sensor for antibiotics in water samples by using electrophoretic preconcentration and SERS (Fig. 4). The AgNP-graphene composites were prepared and deposited on a screen-printed electrode, and then the antibiotic molecules were adsorbed onto the electrode driven by electric field and the $\pi-\pi$ interactions with graphene. Under the multiple signal-amplification mechanisms, four model antibiotics could be detected at sub-nM level within $10 \mathrm{~min}$, making this method suitable for on-site detection.

Zhu et al. [133] used SERS to detect MC-LR in water based on the assembly of AuNRs. The LOD for MC-LR was $5 \mathrm{ng} / \mathrm{L}$, which was at the same level as that with optical detection [124]. To improve the selectivity, Fang et al. [134] used $\beta$-CD to modify the Au-Ag-coated silica-nanosphere array as a SERS substrate in analysis of several endocrine disruptor chemicals (EDCs), and the LODs (300 nM-3.0 $\mu \mathrm{M}$ ) were enhanced by $10-100$ fold compared with those obtained without $\beta-C D$.

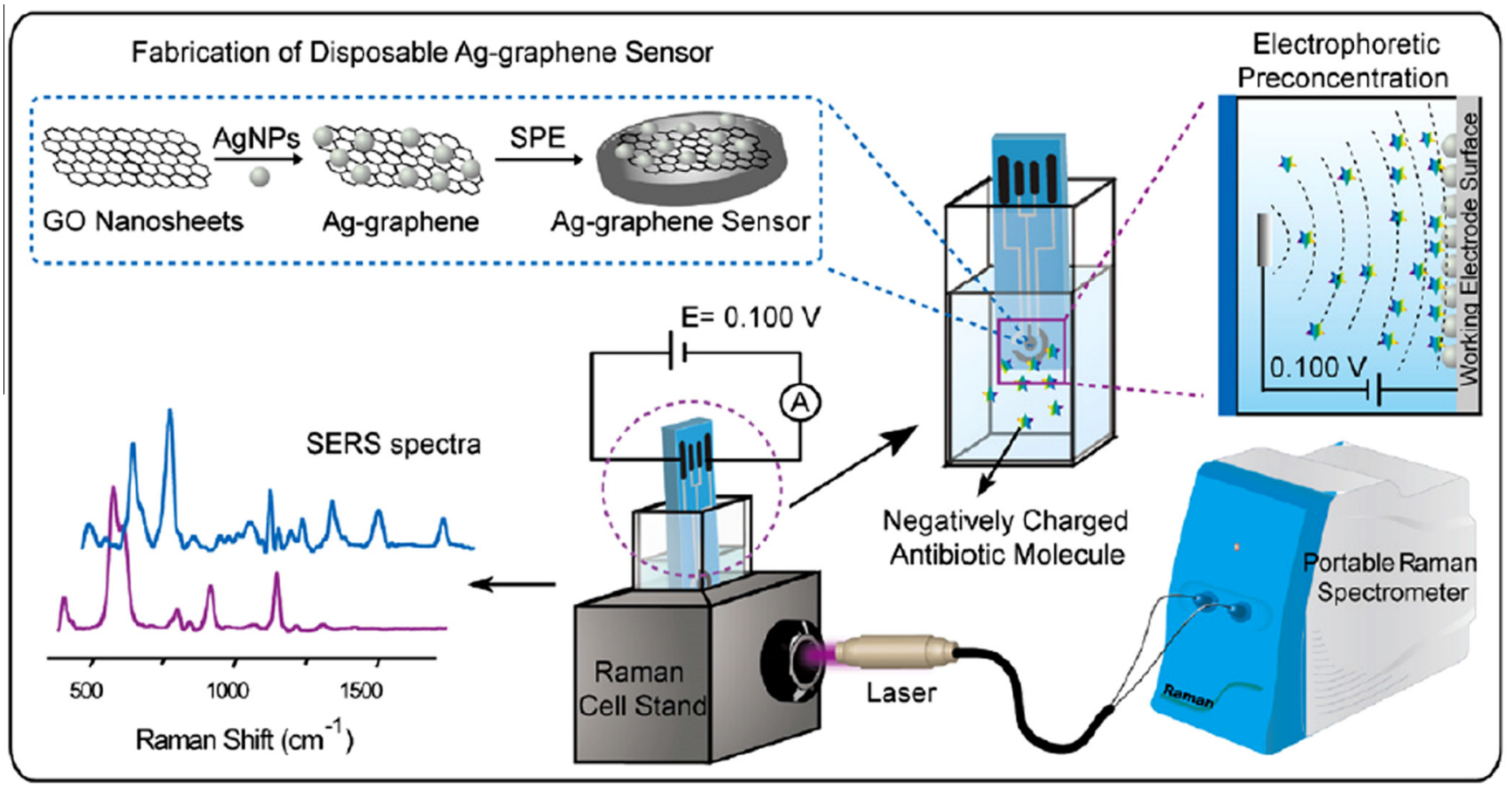

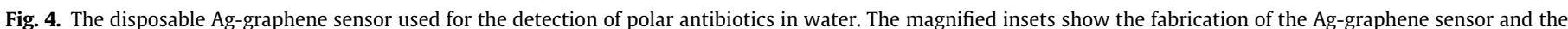

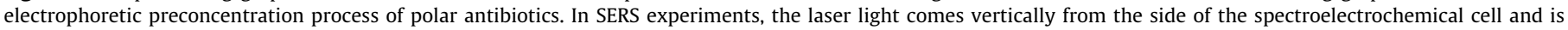
focused on the Ag-graphene sensor [132] (Reprinted with permission from Elsevier). 
He et al. [135] prepared a dendritic Ag nanosubstrate for SERS detection of antibiotics (i.e., enrofloxacin, ciprofloxacin, and chloramphenicol). The Ag dendrites showed a signal-enhancement factor of $\sim 10^{4}$ compared to an Au film substrate, yielding an LOD of $20 \mathrm{ppb}$ for the antibiotics.

\subsection{Matrix-assisted laser desorption/ionization mass spectrometry}

MALDI-time-of-flight (TOF)-MS is a powerful analytical technique for biopolymers and large organic molecules. However, for small molecules, its application is usually restricted due to the strong matrix interference in low-mass region. This problem may be overcome by the application of carbon NMs as MALDI matrices, because carbon NMs have a strong optical absorption ability and high energy-transfer efficiency while producing fewer fragments in the low-mass region, thus offering the opportunity for rapid analysis of small molecules of pollutants by MALDI-TOF MS.

Hu et al. [136] studied the possibility of using CNTs as a matrix in MALDI-TOF-MS for analysis of low-mass chemicals. Different kinds of environmental pollutants (including BPA and BDE-209) were examined.

Liu et al. [137] used graphene and GO as both extractor and matrix for enrichment and detection of tetracyclines in milk. They found that no background inference was caused by graphene or GO matrix, and the LODs were as low as $2 \mathrm{nM}$.

Kawasaki et al. [138] prepared graphene-coated magnetic CoNPs functionalized with benzylamine groups for the enrichment and detection of some ECPs (e.g., BPA, PFCs, and drugs). This method provided high sensitivity for the analytes (e.g., the LOD of perfluorooctanesulfonic acid (PFOS) was $\sim 0.1 \mathrm{ng} / \mathrm{L}$ ).

Recently, Liu et al. [139] reported a simple method to enhance the performance of graphene matrix by mild oxidation of chemically converted graphene with diluted nitric acid. This method can produce low-defect graphene material with good water dispersibility. When this material was used as a matrix in MALDI-TOF MS, higher signals were obtained than using unoxidized graphene, $\mathrm{GO}$, and conventional organic matrices in detection of several typical environmental pollutants (pentachlorophenol, estradiol, PBDE, and PFOS).

\subsection{Other techniques}

Besides the aspects mentioned above, NMs have also been applied in some other techniques to facilitate the analysis of ECPs. In capillary electrophoresis and microchip electrophoresis, NMs were used as stationary phases or buffer additives to enhance the separation or detection. For example, Wang et al. [140] reported an open-tubular capillary electrochromatography method using GO as a stationary phase. The separation of six NSAIDs was greatly enhanced by GO.

Noh et al. [141] developed a microchip for the analysis of endocrine disruptors. Addition of AuNPs in the preconcentration and separation buffers could enhance the analytical sensitivity by $\sim 200$-fold.

Frasconi et al. [142] reported a novel surface-plasmon resonance (SPR) method for detection in milk of three antibiotics having vicinal diol functionalities (neomycin, kanamycin, and streptomycin). A special "MIP" for the analytes was prepared by electropolymerizing the AuNPs functionalized with thioaniline groups and (mercaptophenyl)boronic acid. Upon extraction by this AuNPs-embedded MIP and sensing by SPR, high sensitivity for the antibiotics was obtained (LOD $200 \mathrm{fM}-2.00 \mathrm{pM}$ ).

Fernández et al. [143] compared the signal enhancement of SPR by AuNP probes antibody functionalized in different manners for enrofloxacin detection.
Zhao et al. [144] developed an $\mathrm{AgNO}_{3}$-enhanced electrochemiluminescence (ECL) method for detection of a novel flame retardant, TBC, on an AuNP-modified electrode. The ECL signals were dually enhanced by AuNPs and $\mathrm{AgNO}_{3}$, and the LOD for TBBPA was $5.0 \times 10^{-8} \mathrm{M}$.

Jiang et al. [145,146] have developed nanogold-based resonance-scattering spectral methods for the detection of penicillin $\mathrm{G}$ in milk and BPA in consumer products with LODs of $0.78 \mu \mathrm{g} / \mathrm{L}$ and $0.083 \mu \mathrm{g} / \mathrm{L}$, respectively.

Park et al. [147] developed a piezoelectric immunosensor for BPA detection. 2-Methacrolyloxyethyl phosphorylcholine polymeric NPs with a diameter of $200 \mathrm{~nm}$ were used as a mass label to enhance the sensitivity of the assay. With this NP-based signal-enhancement step, the LOD of BPA was $0.01 \mu \mathrm{g} / \mathrm{L}$.

\section{Conclusions and perspectives}

In summary, application of NMs has become one of the most important trends in environmental analysis. NMs are able to play prominent roles in various techniques to improve the sensitivity, enhance the selectivity, simplify the analytical procedures, and increase the analytical throughput, so NMs provide better opportunities to fulfil the urgent demand in analytical methodology for research on ECPs. That is exactly the reason why the application of NMs in analysis of ECPs has gained rapid growth in recent years. Undoubtedly, NMs have a bright future in this area.

At the same time, it must be realized that the application of NMs is still in its infancy, and there still are some problems or challenges associated with the analysis of ECPs, as follows.

(1) The application of NMs is still limited to a small number of pollutants, and many important pollutants have not benefited from NMs. Furthermore, new pollutants continue to be identified in the environment, resulting in increasing demand on analytical methodology, so more efforts should be made to expand the application of NMs to more types of environmental pollutant, especially newly discovered chemicals.

(2) The novel properties of NMs are a two-edged sword. The same properties that generate exciting benefits in use may also pose significant adverse health effects. For example, the tiny size and strong adsorbing ability of NMs also allow their penetration through skin and cell membrane and strong interaction with biomolecules. As a result, NMs themselves are listed as a class of ECPs. For every NM, careful environment, health and safety evaluation should be performed along with study of its application.

(3) The technology of synthesis is still an obstacle hindering the wide application of NMs, as it is expected to achieve higher production yield and lower production cost of NMs. More importantly, for analytical applications, especially analysis of trace amounts of environmental pollutants, high-quality NMs with higher purity, better monodispersity, and higher stability in long-term use are desired to obtain reliable, reproducible analytical results.

(4) More efforts should be made to explore further the potential of NMs in analysis of complex samples, beyond water samples. Since environmental samples include a great variety of extremely complex matrices, many challenges remain for analytical scientists. For this purpose, more robust NMs with improved selectivity towards the analytes and strong anti-interference to the sample matrix should be developed.

(5) Compared to application in classical techniques as an alternative to conventional materials, it would be more interesting to combine NMs with emerging microtechnology and nanotechnology (e.g., microextraction, nanofluidics, and 
nanoelectrode array) to tackle current analytical problems. It is rational to envision a revolution in environmental analysis caused by NMs in the near future.

\section{Acknowledgements}

The authors acknowledge financial support from the National Basic Research Program of China (No. 2009CB421605) and the National Natural Science Foundation of China (No. 21107120, 21377141).

\section{References}

[1] C.G. Daughton, "Emerging" chemicals as pollutants in the environment: a 21st century perspective, Renew. Resour. J. 23 (2005) 6-23.

[2] M. la Farre, S. Perez, L. Kantiani, D. Barcelo, Fate and toxicity of emerging pollutants, their metabolites and transformation products in the aquatic environment, Trends Anal. Chem. 27 (2008) 991-1007.

[3] T. Deblonde, C. Cossu-Leguille, P. Hartemann, Emerging pollutants in wastewater: a review of the literature, Int. J. Hyg. Environ. Health 214 (2011) 442-448.

[4] S.D. Richardson, T.A. Ternes, Water analysis: emerging contaminants and current issues, Anal. Chem. 83 (2011) 4614-4648.

[5] C. Buzea, I.I. Pacheco, K. Robbie, Nanomaterials and nanoparticles: Sources and toxicity, Biointerphases 2 (2007) Mr17-Mr71.

[6] S.J. Klaine, P.J.J. Alvarez, G.E. Batley, T.F. Fernandes, R.D. Handy, D.Y. Lyon, S. Mahendra, M.J. McLaughlin, J.R. Lead, Nanomaterials in the environment: behavior, fate, bioavailability, and effects, Environ. Toxicol. Chem. 27 (2008) 1825-1851.

[7] M. Farre, K. Gajda-Schrantz, L. Kantiani, D. Barcelo, Ecotoxicity and analysis of nanomaterials in the aquatic environment, Anal. Bioanal. Chem. 393 (2009) 81-95.

[8] S. Sharifi, S. Behzadi, S. Laurent, M.L. Forrest, P. Stroeve, M. Mahmoudi, Toxicity of nanomaterials, Chem. Soc. Rev. 41 (2012) 2323-2343.

[9] S. Park, R.S. Ruoff, Chemical methods for the production of graphenes, Nat. Nanotechnol. 4 (2009) 217-224.

[10] M. Pumera, A. Ambrosi, E.L.K. Chng, Impurities in graphenes and carbon nanotubes and their influence on the redox properties, Chem. Sci. 3 (2012) 3347-3355.

[11] A. Ambrosi, C.K. Chua, B. Khezri, Z. Sofer, R.D. Webster, M. Pumera, Chemically reduced graphene contains inherent metallic impurities present in parent natural and synthetic graphite, Proc. Natl. Acad. Sci. USA 109 (2012) 1289912904.

[12] A.H. Lu, E.L. Salabas, F. Schuth, Magnetic nanoparticles: Synthesis, protection, functionalization, and application, Angew. Chem. Int. Ed. 46 (2007) 1222 1244.

[13] R. Gill, M. Zayats, I. Willner, Semiconductor quantum dots for bioanalysis, Angew. Chem. Int. Ed. 47 (2008) 7602-7625

[14] Y.Q. Cai, G.B. Jiang, J.F. Liu, Q.X. Zhou, Multiwalled carbon nanotubes as a solid-phase extraction adsorbent for the determination of bisphenol A, 4-nnonylphenol, and 4-tert-octylphenol, Anal. Chem. 75 (2003) 2517-2521.

[15] Y.Q. Cai, G.B. Jiang, J.F. Liu, Q.X. Zhou, Multi-walled carbon nanotubes packed cartridge for the solid-phase extraction of several phthalate esters from water samples and their determination by high performance liquid chromatography, Anal. Chim. Acta 494 (2003) 149-156.

[16] S. Dahane, M.D.G. Garcia, M.J.M. Bueno, A.U. Moreno, M.M. Galera, A. Derdour, Determination of drugs in river and wastewaters using solid-phase extraction by packed multi-walled carbon nanotubes and liquid chromatographyquadrupole-linear ion trap-mass spectrometry, J. Chromatogr. A 1297 (2013) 17-28.

[17] G.Z. Fang, J.X. He, S. Wang, Multiwalled carbon nanotubes as sorbent for online coupling of solid-phase extraction to high-performance liquid chromatography for simultaneous determination of 10 sulfonamides in eggs and pork, J. Chromatogr. A 1127 (2006) 12-17.

[18] B. Suadrez, B. Santos, B.M. Simonet, S. Cardenas, M. Valcarcel, Solid-phase extraction-capillary electrophoresis-mass spectrometry for the determination of tetracyclines residues in surface water by using carbon nanotubes as sorbent material, J. Chromatogr. A 1175 (2007) 127-132.

[19] X.L. Hou, Y.L. Wu, T. Yang, X.D. Du, Multi-walled carbon nanotubes-dispersive solid-phase extraction combined with liquid chromatography-tandem mass spectrometry for the analysis of 18 sulfonamides in pork, J. Chromatogr. B 929 (2013) 107-115.

[20] M.M. Li, X.G. Liu, F.S. Dong, J. Xu, Z.Q. Kong, Y.B. Li, Y.Q. Zheng, Simultaneous determination of cyflumetofen and its main metabolite residues in samples of plant and animal origin using multi-walled carbon nanotubes in dispersive solid-phase extraction and ultrahigh performance liquid chromatographytandem mass spectrometry, J. Chromatogr. A 1300 (2013) 95-103.

[21] R. Su, X.H. Wang, X. Xu, Z.M. Wang, D. Li, X. Zhao, X.Y. Li, H.Q. Zhang, A.M. Yu, Application of multiwall carbon nanotubes-based matrix solid phase dispersion extraction for determination of hormones in butter by gas chromatography mass spectrometry, J. Chromatogr. A 1218 (2011) 50475054.

[22] J.X. Wang, D.Q. Jiang, Z.Y. Gu, X.P. Yan, Multiwalled carbon nanotubes coated fibers for solid-phase microextraction of polybrominated diphenyl ethers in water and milk samples before gas chromatography with electron-capture detection, J. Chromatogr. A 1137 (2006) 8-14.

[23] X.X. Ma, Q.L. Li, D.X. Yuan, Determination of endocrine-disrupting compounds in water by carbon nanotubes solid-phase microextraction fiber coupled online with high performance liquid chromatography, Talanta 85 (2011) 2212-2217.

[24] W.Y. Zhang, Y. Sun, C.Y. Wu, J. Xing, J.Y. Li, Polymer-functionalized singlewalled carbon nanotubes as a novel sol-gel solid-phase micro-extraction coated fiber for determination of polybrominated diphenyl ethers in water samples with gas chromatography-electron capture detection, Anal. Chem. 81 (2009) 2912-2920.

[25] Z. Es'haghi, Z. Rezaeifar, G.H. Rounaghi, Z.A. Nezhadi, M.A. Golsefidi, Synthesis and application of a novel solid-phase microextraction adsorbent: hollow fiber supported carbon nanotube reinforced sol-gel for determination of phenobarbital, Anal. Chim. Acta 689 (2011) 122-128.

[26] A. Sarafraz-Yazdi, A. Amiri, G. Rounaghi, H. Eshtiagh-Hosseini, Determination of non-steroidal anti-inflammatory drugs in water samples by solid-phase microextraction based sol-gel technique using poly(ethylene glycol) grafted multi-walled carbon nanotubes coated fiber, Anal. Chim. Acta 720 (2012) 134-141.

[27] A. Sarafraz-Yazdi, S. Sepehr, Z. Es'haghi, H.P. Moghadam, Application of solgel based poly(ethylene glycol)/multiwalled carbon nanotubes coated fiber for SPME of methyl tert-butyl ether in environmental water samples, Chromatographia 72 (2010) 923-931.

[28] A. Sarafraz-Yazdi, A. Amiri, G. Rounaghi, H. Eshtiagh-Hosseini, Headspace solid-phase microextraction using poly(ethylene glycol) grafted multi-walled carbon nanotube fibers for the determination of methyl tert-butyl ether in water samples, Anal. Methods 4 (2012) 3701-3707.

[29] X.Y. Song, Y.P. Shi, J. Chen, A novel extraction technique based on carbon nanotubes reinforced hollow fiber solid/liquid microextraction for the measurement of piroxicam and diclofenac combined with high performance liquid chromatography, Talanta 100 (2012) 153-161.

[30] K.S. Hasheminasab, A.R. Fakhari, A. Shahsavani, H. Ahmar, A new method for the enhancement of electromembrane extraction efficiency using carbon nanotube reinforced hollow fiber for the determination of acidic drugs in spiked plasma, urine, breast milk and wastewater samples, J. Chromatogr. A 1285 (2013) 1-6.

[31] A. Sarafraz-Yazdi, M.R. Abedi, Z. Es'haghi, Pre-concentration and determination of beta-blockers using carbon nanotube-assisted pseudostirbar hollow fiber solid-/liquid-phase microextraction and highperformance liquid chromatography with fluorescence detection, J. Liq. Chromatogr. Rel. Technol. 36 (2013) 750-769.

[32] Q. Liu, J.B. Shi, G.B. Jiang, Application of graphene in analytical sample preparation, Trends Anal. Chem. 37 (2012) 1-11.

[33] N.S. Ye, P.Z. Shi, Q. Wang, J. Li, Graphene as solid-phase extraction adsorbent for CZE determination of sulfonamide residues in meat samples, Chromatographia 76 (2013) 553-557.

[34] Q. Liu, J.B. Shi, J.T. Sun, T. Wang, L.X. Zeng, G.B. Jiang, Graphene and graphene oxide sheets supported on silica as versatile and high-performance adsorbents for solid-phase extraction, Angew. Chem. Int. Ed. 50 (2011) 5913-5917.

[35] Y. Yu, L.S. Wu, Application of graphene for the analysis of pharmaceuticals and personal care products in wastewater, Anal. Bioanal. Chem. 405 (2013) 4913-4919.

[36] H. Zhang, H.K. Lee, Plunger-in-needle solid-phase microextraction with graphene-based sol-gel coating as sorbent for determination of polybrominated diphenyl ethers, J. Chromatogr. A 1218 (2011) 4509-4516.

[37] O. Liu, M. Cheng, Y. Long M. Yu, T. Wang, G. Jiang Graphenized pencil lead fiber: facile preparation and application in solid-phase microextraction, J. Chromatogr. A 1325 (2014) 1-7.

[38] Q. Liu, J.B. Shi, J.T. Sun, T. Wang, L.X. Zeng, N.L. Zhu, G.B. Jiang, Grapheneassisted matrix solid-phase dispersion for extraction of polybrominated diphenyl ethers and their methoxylated and hydroxylated analogs from environmental samples, Anal. Chim. Acta 708 (2011) 61-68.

[39] Q.L. Li, M.H.W. Lam, R.S.S. Wu, B.W. Jiang, Rapid magnetic-mediated solidphase extraction and pre-concentration of selected endocrine disrupting chemicals in natural waters by poly(divinylbenzene-co-methacrylic acid) coated $\mathrm{Fe}_{3} \mathrm{O}_{4}$ core-shell magnetite microspheres for their liquid chromatography-tandem mass spectrometry determination, J. Chromatogr. A 1217 (2010) 1219-1226.

[40] Q. Gao, D. Luo, M. Bai, Z.W. Chen, Y.Q. Feng, Rapid determination of estrogens in milk samples based on magnetite nanoparticles/polypyrrole magnetic solid-phase extraction coupled with liquid chromatography-tandem mass spectrometry, J. Agric. Food Chem. 59 (2011) 8543-8549.

[41] L. Sun, X. Sun, X.B. Du, Y.S. Yue, L.G. Chen, H.Y. Xu, Q.L. Zeng, H. Wang, L. Ding, Determination of sulfonamides in soil samples based on alumina-coated magnetite nanoparticles as adsorbents, Anal. Chim. Acta 665 (2010) 185-192.

[42] H.Y. Niu, Y.X. Wang, X.L. Zhang, Z.F. Meng, Y.Q. Cai, Easy synthesis of surfacetunable carbon-encapsulated magnetic nanoparticles: adsorbents for selective isolation and preconcentration of organic pollutants, ACS Appl. Mater. Interfaces 4 (2012) 286-295.

[43] I.P. Roman, A. Chisvert, A. Canals, Dispersive solid-phase extraction based on oleic acid-coated magnetic nanoparticles followed by gas chromatography- 
mass spectrometry for UV-filter determination in water samples, J. Chromatogr. A 1218 (2011) 2467-2475.

[44] X.L. Zhang, H.Y. Niu, Y.Y. Pan, Y.L. Shi, Y.Q. Cai, Chitosan-coated octadecylfunctionalized magnetite nanoparticles: preparation and application in extraction of trace pollutants from environmental water samples, Anal. Chem. 82 (2010) 2363-2371.

[45] Z. Yan, Y. Cai, G. Zhu, J. Yuan, L. Tu, C. Chen, S. Yao, Synthesis of 3fluorobenzoyl chloride functionalized magneticsorbent for highly efficient enrichment of perfluorinated compounds from river water samples, J. Chromatogr. A 1321 (2013) 21-29.

[46] L. Yang, W.J. Yu, X.M. Yan, C.H. Deng, Decyl-perfluorinated magnetic mesoporous microspheres for extraction and analysis perfluorinated compounds in water using ultrahigh-performance liquid chromatographymass spectrometry, J. Sep. Sci. 35 (2012) 2629-2636.

[47] H. Bagheri, O. Zandi, A. Aghakhani, Extraction of fluoxetine from aquatic and urine samples using sodium dodecyl sulfate-coated iron oxide magnetic nanoparticles followed by spectrofluorimetric determination, Anal. Chim. Acta 692 (2011) 80-84.

[48] Y. Moliner-Martinez, A. Ribera, E. Coronado, P. Campins-Falco, Preconcentration of emerging contaminants in environmental water samples by using silica supported $\mathrm{Fe}_{3} \mathrm{O}_{4}$ magnetic nanoparticles for improving mass detection in capillary liquid chromatography, J. Chromatogr. A 1218 (2011) 2276-2283.

[49] S.R. Yazdinezhad, A. Ballesteros-Gomez, L. Lunar, S. Rubio, Single-step extraction and cleanup of bisphenol $A$ in soft drinks by hemimicellar magnetic solid phase extraction prior to liquid chromatography/tandem mass spectrometry, Anal. Chim. Acta 778 (2013) 31-37.

[50] Y.S. Ji, X.Y. Liu, M. Guan, C.D. Zha, H.Y. Huang, H.X. Zhang, C.M. Wang, Preparation of functionalized magnetic nanoparticulate sorbents for rapid extraction of biphenolic pollutants from environmental samples, J. Sep. Sci. 32 (2009) 2139-2145.

[51] Y. Jiao, L. Ding, S. Fu, S. Zhu, H. Li, L. Wang, Determination of bisphenol A, bisphenol $\mathrm{F}$ and their diglycidyl ethers in environmental water by solid phase extraction using magnetic multiwalled carbon nanotubes followed by GCMS/MS, Anal. Methods 4 (2012) 291-298.

[52] Y. Jiao, S. Fu, L. Ding, Q. Gong, S. Zhu, L. Wang, H. Li, Determination of trace leaching phthalate esters in water by magnetic solid phase extraction based on magnetic multi-walled carbon nanotubes followed by GC-MS/MS, Anal. Methods 4 (2012) 2729-2734.

[53] Y. Xu, J. Ding, H. Chen, Q. Zhao, J. Hou, J. Yan, H. Wang, L. Ding, N. Ren, Fast determination of sulfonamides from egg samples using magnetic multiwalled carbon nanotubes as adsorbents followed by liquid chromatography-tandem mass spectrometry, Food Chem. 140 (2013) 83-90.

[54] W. Wang, R. Ma, Q. Wu, C. Wang, Z. Wang, Fabrication of magnetic microsphere-confined graphene for the preconcentration of some phthalate esters from environmental water and soybean milk samples followed by their determination by HPLC, Talanta 109 (2013) 133-140.

[55] Q. Wu, M. Liu, X. Ma, W. Wang, C. Wang, X. Zang, Z. Wang, Extraction of phthalate esters from water and beverages using a graphene-based magnetic nanocomposite prior to their determination by HPLC, Microchim. Acta 177 (2012) 23-30.

[56] Y.B. Luo, Q.W. Yu, B.F. Yuan, Y.Q. Feng, Fast microextraction of phthalate acid esters from beverage, environmental water and perfume samples by magnetic multi-walled carbon nanotubes, Talanta 90 (2012) 123-131.

[57] Y.B. Luo, Z.G. Shi, Q. Gao, Y.Q. Feng, Magnetic retrieval of graphene: extraction of sulfonamide antibiotics from environmental water samples, J. Chromatogr. A 1218 (2011) 1353-1358.

[58] R. Gao, X. Kong, F. Su, X. He, L. Chen, Y. Zhang, Synthesis and evaluation of molecularly imprinted core-shell carbon nanotubes for the determination of triclosan in environmental water samples, J. Chromatogr. A 1217 (2010) 8095-8102.

[59] Y. Liu, Y. Huang, J. Liu, W. Wang, G. Liu, R. Zhao, Superparamagnetic surface molecularly imprinted nanoparticles for water-soluble pefloxacin mesylate prepared via surface initiated atom transfer radical polymerization and its application in egg sample analysis, J. Chromatogr. A 1246 (2012) 15-21.

[60] Z. Lin, W. Cheng, Y. Li, Z. Liu, X. Chen, C. Huang, A novel superparamagnetic surface molecularly imprinted nanoparticle adopting dummy template: an efficient solid-phase extraction adsorbent for bisphenol A, Anal. Chim. Acta 720 (2012) 71-76.

[61] F. Wei, X. Liu, M. Zhai, Z. Cai, G. Xu, J. Yang, S. Du, Q. Hu, Molecularly imprinted nanosilica solid-phase extraction for bisphenol $\mathrm{A}$ in fish samples, Food Anal. Methods 6 (2013) 415-420.

[62] Q. Liu, J. Shi, T. Wang, F. Guo, L. Liu, G. Jiang, Hemimicelles/admicelles supported on magnetic graphene sheets for enhanced magnetic solid-phase extraction, J. Chromatogr. A 1257 (2012) 1-8.

[63] X. Zhao, Y. Shi, Y. Cai, S. Mou, Cetyltrimethylammonium bromide-coated magnetic nanoparticles for the preconcentration of phenolic compounds from environmental water samples, Environ. Sci. Technol. 42 (2008) 12011206.

[64] X. Zhao, Y. Shi, T. Wang, Y. Cai, G. Jiang, Preparation of silica-magnetite nanoparticle mixed hemimicelle sorbents for extraction of several typical phenolic compounds from environmental water samples, J. Chromatogr. A 1188 (2008) 140-147.

[65] H. Niu, Y. Cai, Y. Shi, F. Wei, S. Mou, G. Jiang, Cetyltrimethylammonium bromide-coated titanate nanotubes for solid-phase extraction of phthalate esters from natural waters prior to high-performance liquid chromatography analysis, J. Chromatogr. A 1172 (2007) 113-120.

[66] P.-L. Liu, Y.-P. Xu, P. Zheng, H.-W. Tong, Y.-X. Liu, Z.-G. Zha, Q.-D. Sua, S.-M. Liu, Mesoporous silica-coated magnetic nanoparticles for mixed hemimicelles solid-phase extraction of phthalate esters in environmental water samples with liquid chromatographic analysis, J. Chin. Chem. Soc. 60 (2013) 53-62.

[67] X. Wang, J. Liu, A. Liu, Q. Liu, X. Du, G. Jiang, Preparation and evaluation of mesoporous cellular foams coating of solid-phase microextraction fibers by determination of tetrabromobisphenol A, tetrabromobisphenol S and related compounds, Anal. Chim. Acta 753 (2012) 1-7.

[68] Q. Xu, S.-Y. Wu, M. Wang, X.-Y. Yin, Z.-Y. Wen, W.-N. Ge, Z.-Z. Gu, Electrospun Nylon6 nanofibrous membrane as SPE adsorbent for the enrichment and determination of three estrogens in environmental water samples, Chromatographia 71 (2010) 487-492.

[69] Q. Xu, X. Yin, S. Wu, M. Wang, Z. Wen, Z. Gu, Determination of phthalate esters in water samples using Nylon6 nanofibers mat-based solid-phase extraction coupled to liquid chromatography, Microchim. Acta 168 (2010) 267-275.

[70] M.H. Banitaba, S.S. Davarani, A. Pourahadi, Solid-phase microextraction of phthalate esters from aqueous media by electrophoretically deposited $\mathrm{TiO}_{2}$ nanoparticles on a stainless steel fiber, J. Chromatogr. A 1283 (2013) 1-8.

[71] D. Vega, L. Agui, A. Gonzalez-Cortes, R. Yanez-Sedeno, J.M. Pingarron, Electrochemical detection of phenolic estrogenic compounds at carbon nanotube-modified electrodes, Talanta 71 (2007) 1031-1038.

[72] E.J. Parra, G.A. Crespo, J. Riu, A. Ruiz, F.X. Rius, Ion-selective electrodes using multi-walled carbon nanotubes as ion-to-electron transducers for the detection of perchlorate, Analyst 134 (2009) 1905-1910.

[73] H.T. Wang, H.M. Zhao, X. Quan, S. Chen, Electrochemical determination of tetracycline using molecularly imprinted polymer modified carbon nanotube-gold nanoparticles electrode, Electroanalysis 23 (2011) 18631869.

[74] F.C. Moraes, I. Cesarino, V. Cesarino, L.H. Mascaro, S.A.S. Machado, Carbon nanotubes modified with antimony nanoparticles: a novel material for electrochemical sensing, Electrochim. Acta 85 (2012) 560-565.

[75] X.L. Sun, L. Guan, H. Shi, J. Ji, Y.Z. Zhang, Z.J. Li, Determination of microcystinLR with a glassy carbon impedimetric immunoelectrode modified with an ionic liquid and multiwalled carbon nanotubes, Microchim. Acta 180 (2013) $75-83$.

[76] Z.X. Zheng, Y.L. Du, Z.H. Wang, Q.L. Feng, C.M. Wang, Pt/graphene-CNTs nanocomposite based electrochemical sensors for the determination of endocrine disruptor bisphenol A in thermal printing papers, Analyst 138 (2013) 693-701.

[77] H.S. Yin, Y.L. Zhou, J. Xu, S.Y. Ai, L. Cui, L.S. Zhu, Amperometric biosensor based on tyrosinase immobilized onto multiwalled carbon nanotubes-cobalt phthalocyanine-silk fibroin film and its application to determine bispheno A, Anal. Chim. Acta 659 (2010) 144-150.

[78] X.Q. Liu, H.Q. Feng, X.H. Liu, D.K.Y. Wong, Electrocatalytic detection of phenolic estrogenic compounds at NiTPPS|carbon nanotube composite electrodes, Anal. Chim. Acta 689 (2011) 212-218.

[79] L. Zhou, D.J. Li, L. Gai, J.P. Wang, Y.B. Li, Electrochemical aptasensor for the detection of tetracycline with multi-walled carbon nanotubes amplification, Sensors Actuat. B: Chem. 162 (2012) 201-208.

[80] Y. Gao, Y. Cao, D.G. Yang, X.J. Luo, Y.M. Tang, H.M. Li, Sensitivity and selectivity determination of bisphenol A using SWCNT-CD conjugate modified glassy carbon electrode, J. Hazard. Mater. 199 (2012) 111-118.

[81] Y.G. Li, Y. Gao, Y. Cao, H.M. Li, Electrochemical sensor for bisphenol A determination based on MWCNT/melamine complex modified GCE, Sensors Actuat. B: Chem. 171 (2012) 726-733.

[82] F.C. Moraes, T.A. Silva, I. Cesarino, S.A.S. Machado, Effect of the surface organization with carbon nanotubes on the electrochemical detection of bisphenol A, Sensors Actuat. B: Chem. 177 (2013) 14-18.

[83] Q.J. Wan, H. Cai, Y. Liu, H.T. Song, H.L. Liao, S.T. Liu, N.J. Yang, Graphene nanoplatelets: electrochemical properties and applications for oxidation of endocrine-disrupting chemicals, Chem. Eur. J. 19 (2013) 3483-3489.

[84] S. Pruneanu, F. Pogacean, A.R. Biris, S. Ardelean, V. Canpean, G. Blanita, E. Dervishi, A.S. Biris, Novel graphene-gold nanoparticle modified electrodes for the high sensitivity electrochemical spectroscopy detection and analysis of carbamazepine, J. Phys. Chem. C 115 (2011) 23387-23394.

[85] R.Y. Li, Q.F. Xia, Z.J. Li, X.L. Sun, J.K. Liu, Electrochemical immunosensor for ultrasensitive detection of microcystin-LR based on graphene-gold nanocomposite/functional conducting polymer/gold nanoparticle/ionic liquid composite film with electrodeposition, Biosens. Bioelectron. 44 (2013) 235-240.

[86] A. Gutes, B.Y. Lee, C. Carraro, W. Mickelson, S.W. Lee, R. Maboudian, Impedimetric graphene-based biosensors for the detection of polybrominated diphenyl ethers, Nanoscale 5 (2013) 6048-6052.

[87] Y.X. Zhang, Y.X. Cheng, Y.Y. Zhou, B.Y. Li, W. Gu, X.H. Shi, Y.Z. Xian, Electrochemical sensor for bisphenol A based on magnetic nanoparticles decorated reduced graphene oxide, Talanta 107 (2013) 211-218.

[88] S.J. Yu, Q. Wei, B. Du, D. Wu, H. Li, L.G. Yan, H.M. Ma, Y. Zhang, Label-free immunosensor for the detection of kanamycin using $\mathrm{Ag} @ \mathrm{Fe}_{3} \mathrm{O}_{4}$ nanoparticles and thionine mixed graphene sheet, Biosens. Bioelectron. 48 (2013) 224-229.

[89] L.D. Wu, D.H. Deng, J. Jin, X.B. Lu, J.P. Chen, Nanographene-based tyrosinase biosensor for rapid detection of bisphenol A, Biosens. Bioelectron. 35 (2012) 193-199. 
[90] Y. Qu, M. Ma, Z.G. Wang, G.Q. Zhan, B.H. Li, X. Wang, H.F. Fang, H.J. Zhang, C.Y $\mathrm{Li}$, Sensitive amperometric biosensor for phenolic compounds based on graphene-silk peptide/tyrosinase composite nanointerface, Biosens. Bioelectron. 44 (2013) 85-88.

[91] H.X. Fan, Y. Li, D. Wu, H.M. Ma, K.X. Mao, D.W. Fan, B. Du, H. Li, Q. Wei, Electrochemical bisphenol A sensor based on N-doped graphene sheets, Anal. Chim. Acta 711 (2012) 24-28.

[92] Q.X. Wang, Y.H. Wang, S.Y. Liu, L.H. Wang, F. Gao, F. Gao, W. Sun Voltammetric detection of bisphenol a by a chitosan-graphene composite modified carbon ionic liquid electrode, Thin Solid Films 520 (2012) 44594464.

[93] H.J. Chen, Z.H. Zhang, R. Cai, X.Q. Kong, X. Chen, Y.N. Liu, S.Z. Yao, Molecularly imprinted electrochemical sensor based on a reduced graphene modified carbon electrode for tetrabromobisphenol A detection, Analyst 138 (2013) 2769-2776.

[94] Q. Wei, Y.F. Zhao, B. Du, D. Wu, Y.Y. Cai, K.X. Mao, H. Li, C.X. Xu, Nanoporous PtRu alloy enhanced nonenzymatic immunosensor for ultrasensitive detection of microcystin-LR, Adv. Funct. Mater. 21 (2011) 4193-4198.

[95] J. Zhang, J.P. Lei, C.L. Xu, L. Ding, H.X. Ju, Carbon nanohorn sensitized electrochemical immunosensor for rapid detection of microcystin-LR, Anal. Chem. 82 (2010) 1117-1122.

[96] G.F. Xu, L.S. Gong, H. Dai, X.H. Li, S.P. Zhang, S.Y. Lu, Y.Y. Lin, J.H. Chen, Y.J. Tong, G.N. Chen, Electrochemical bisphenol A sensor based on carbon nanohorns, Anal. Methods 5 (2013) 3328-3333.

[97] W. Xia, Y.Y. Li, Y.J. Wan, T.A. Chen, J. Wei, Y. Lin, S.Q. Xu, Electrochemical biosensor for estrogenic substance using lipid bilayers modified by $\mathrm{Au}$ nanoparticles, Biosens. Bioelectron. 25 (2010) 2253-2258.

[98] Y. Zhu, P. Chandra, K.M. Song, C. Ban, Y.B. Shim, Label-free detection of kanamycin based on the aptamer-functionalized conducting polymer/gold nanocomposite, Biosens. Bioelectron. 36 (2012) 29-34.

[99] J.D. Huang, X.M. Zhang, S. Liu, Q. Lin, X.R. He, X.R. Xing, W.J. Lian, Electrochemical sensor for bisphenol A detection based on molecularly imprinted polymers and gold nanoparticles, J. Appl. Electrochem. 41 (2011) 1323-1328.

[100] R.S.J. Alkasir, M. Ganesana, Y.H. Won, L. Stanciu, S. Andreescu, Enzyme functionalized nanoparticles for electrochemical biosensors: a comparative study with applications for the detection of bisphenol A, Biosens. Bioelectron. 26 (2010) 43-49.

[101] E. Valera, A. Muriano, M.I. Pividori, F. Sanchez-Baeza, M.P. Marco, Development of a Coulombimetric immunosensor based on specific antibodies labeled with CdS nanoparticles for sulfonamide antibiotic residues analysis and its application to honey samples, Biosens. Bioelectron. 43 (2013) 211-217.

[102] B.J. Lu, M.C. Liu, H.J. Shi, X.F. Huang, G.H. Zhao, A novel photoelectrochemical sensor for bisphenol A with high sensitivity and selectivity based on surface molecularly imprinted polypyrrole modified $\mathrm{TiO}_{2}$ nanotubes, Electroanalysis 25 (2013) 771-779.

[103] A. Qurashi, J.A. Rather, K. De Wael, B. Merzougui, N. Tabet, M. Faiz, Rapid microwave synthesis of high aspect-ratio $\mathrm{ZnO}$ nanotetrapods for swift bisphenol A detection, Analyst 138 (2013) 4764-4768.

[104] H.S. Yin, Y.L. Zhou, S.Y. Ai, Q.P. Chen, X.B. Zhu, X.G. Liu, L.S. Zhu, Sensitivity and selectivity determination of BPA in real water samples using PAMAM dendrimer and CoTe quantum dots modified glassy carbon electrode, J. Hazard. Mater. 174 (2010) 236-243.

[105] F.G. Wang, J.Q. Yang, K.B. Wu, Mesoporous silica-based electrochemical sensor for sensitive determination of environmental hormone bisphenol A Anal. Chim. Acta 638 (2009) 23-28.

[106] E.R. Carvalho, N.C. Filho, E.C. Venancio, N.O. Osvaldo, L.H.C. Mattoso, L. Martin-Neto, Detection of brominated by-products using a sensor array based on nanostructured thin films of conducting polymers, Sensors 7 (2007) 3258-3271.

[107] X.P. Zhou, Y.H. Meng, H.B. Ma, G.H. Tao, Method for determination of microcystin-leucine-arginine in water samples based on the quenching of the fluorescence of bioconjugates between $\mathrm{CdSe} / \mathrm{CdS}$ quantum dots and microcystin-leucine-arginine antibody, Microchim. Acta 173 (2011) 259 266.

[108] R. Kuang, X. Kuang, S.Y. Pan, X.D. Zheng, J.C. Duan, Y.Q. Duan, Synthesis of cysteamine-coated CdTe quantum dots for the detection of bisphenol A Microchim. Acta 169 (2010) 109-115.

[109] J.X. Chen, F. Xu, H.Y. Jiang, Y.L. Hou, Q.X. Rao, P.J. Guo, S.Y. Ding, A novel quantum dot-based fluoroimmunoassay method for detection of Enrofloxacin residue in chicken muscle tissue, Food Chem. 113 (2009) 1197-1201.

[110] Y.P. Chen, D.N. Wang, Y.M. Yin, L.Y. Wang, X.F. Wang, M.X. Xie, Quantum dots capped with dummy molecularly imprinted film as luminescent sensor for the determination of tetrabromobisphenol A in water and soils, J. Agric. Food Chem. 60 (2012) 10472-10479.

[111] Y. Shi, J.Z. Wu, Y.J. Sun, Y. Zhang, Z.W. Wen, H.C. Dai, H.D. Wang, Z. Li, A graphene oxide based biosensor for microcystins detection by fluorescence resonance energy transfer, Biosens. Bioelectron. 38 (2012) 31-36.

[112] N. Davydov, R. Zairov, A. Mustafina, V. Syakayev, D. Tatarinov, V. Mironov, S Eremin, A. Konovalov, M. Mustafin, Determination of fluoroquinolone antibiotics through the fluorescent response of Eu(III) based nanoparticles fabricated by layer-by-layer technique, Anal. Chim. Acta 784 (2013) 65-71.
[113] H.M. Zhao, S. Gao, M. Liu, Y.Y. Chang, X.F. Fan, X. Quan, Fluorescent assay for oxytetracycline based on a long-chain aptamer assembled onto reduced graphene oxide, Microchim. Acta 180 (2013) 829-835.

[114] M. Liu, H.M. Zhao, S. Chen, H.T. Yu, X. Quan, Colloidal graphene as a transducer in homogeneous fluorescence-based immunosensor for rapid and sensitive analysis of microcystin-LR, Environ. Sci. Technol. 46 (2012) 12567 12574.

[115] H. Font, J. Adrian, R. Galve, M.C. Esevez, M. Castellari, M. Gratacos-Cubarsi, F. Sanchez-Baeza, M.P. Marcot, Immunochemical assays for direct sulfonamide antibiotic detection in milk and hair samples using antibody derivatized magnetic nanoparticles, J. Agric. Food Chem. 56 (2008) 736-743.

[116] J. Huang, H.Q. Liu, H.F. Men, Y.Y. Zhai, Q.H. Xi, Z.L. Zhang, J. Zhang, Z.Z. Yin, L. Li, Molecularly imprinted polymer coating with fluorescence on magnetic particle, Macromol. Res. 21 (2013) 1021-1028.

[117] K.I. Gabrovska, S.I. Ivanova, Y.L. Ivanov, T.I. Godjevargova, Immunofluorescent analysis with magnetic nanoparticles for simultaneous determination of antibiotic residues in milk, Anal. Lett. 46 (2013) 1537-1552.

[118] H.L. Tan, Y. Chen, Silver nanoparticle enhanced fluorescence of europium (III) for detection of tetracycline in milk, Sensors Actuat. B: Chem. 173 (2012) 262-267.

[119] Z.L. Mei, H.Q. Chu, W. Chen, F. Xue, J. Liu, H.N. Xu, R. Zhang, L. Zheng, Ultrasensitive one-step rapid visual detection of bisphenol A in water samples by label-free aptasensor, Biosens. Bioelectron. 39 (2013) 26-30.

[120] K.V. Ragavan, L.S. Selvakumar, M.S. Thakur, Functionalized aptamers as nanobioprobes for ultrasensitive detection of bisphenol-A, Chem. Commun. 49 (2013) 5960-5962.

[121] Y.S. Kim, J.H. Kim, I.A. Kim, S.J. Lee, J. Jurng, M.B. Gu, A novel colorimetric aptasensor using gold nanoparticle for a highly sensitive and specific detection of oxytetracycline, Biosens. Bioelectron. 26 (2010) 1644-1649.

[122] K.M. Song, M. Cho, H. Jo, K. Min, S.H. Jeon, T. Kim, M.S. Han, J.K. Ku, C. Ban, Gold nanoparticle-based colorimetric detection of kanamycin using a DNA aptamer, Anal. Biochem. 415 (2011) 175-181.

[123] M. Takayose, K. Akamatsu, H. Nawafune, T. Murashima, J. Matsui, Colorimetric detection of perfluorooctanoic acid (PFOA) utilizing polystyrene-modified gold nanoparticles, Anal. Lett. 45 (2012) 2856-2864.

[124] L.B. Wang, Y.Y. Zhu, L.G. Xu, W. Chen, H. Kuang, L.Q. Liu, A. Agarwal, C.L. Xu, N.A. Kotov, Side-by-side and end-to-end gold nanorod assemblies for environmental toxin sensing, Angew. Chem. Int. Ed. 49 (2010) 5472-5475.

[125] Y.Y. Zhu, C.L. Qu, H. Kuang, L.G. Xu, L.Q. Liu, Y.F. Hua, L.B. Wang, C.L. Xu, Simple, rapid and sensitive detection of antibiotics based on the side-by-side assembly of gold nanorod probes, Biosens. Bioelectron. 26 (2011) 4387-4392.

[126] Z.L. Mei, Y. Deng, H.Q. Chu, F. Xue, Y.H. Zhong, J.J. Wu, H. Yang, Z.C. Wang, L. Zheng, W. Chen, Immunochromatographic lateral flow strip for on-site detection of bisphenol A, Microchim. Acta 180 (2013) 279-285.

[127] Z.L. Mei, W. Qu, Y. Deng, H.Q. Chu, J.X. Cao, F. Xue, L. Zheng, H.S. El-Nezamic, Y.C. Wu, W. Chen, One-step signal amplified lateral flow strip biosensor for ultrasensitive and on-site detection of bisphenol A (BPA) in aqueous samples, Biosens. Bioelectron. 49 (2013) 457-461.

[128] C. Ruan, W. Wang, B. Gu, Surface-enhanced Raman scattering for perchlorate detection using cystamine-modified gold nanoparticles, Anal. Chim. Acta 567 (2006) 114-120.

[129] W. Wang, C. Ruan, B. Gu, Development of gold-silica composite nanoparticle substrates for perchlorate detection by surface-enhanced Raman spectroscopy, Anal. Chim. Acta 567 (2006) 121-126.

[130] J. Hao, Z. Xu, M.-J. Han, S. Xu, X. Meng, Surface-enhanced Raman scattering analysis of perchlorate using silver nanofilms deposited on copper foils, Colloids Surf. A: Physicochem. Eng. Aspects 366 (2010) 163-169.

[131] J. Hao, M.J. Han, J. Li, X. Meng, Surface modification of silver nanofilms for improved perchlorate detection by surface-enhanced Raman scattering, J. Colloid Interface Sci. 377 (2012) 51-57.

[132] YT. Li, L.L. Qu, D.W. Li, QX. Song, F. Fathi, YT. Long Rapid and sensitive insitu detection of polar antibiotics in water using a disposable Ag-graphene sensor based on electrophoretic preconcentration and surface-enhanced Raman spectroscopy, Biosens. Bioelectron. 43 (2013) 94-100.

[133] Y. Zhu, H. Kuang, L. Xu, W. Ma, C. Peng, Y. Hua, L. Wang, C. Xu, Gold nanorod assembly based approach to toxin detection by SERS, J. Mater. Chem. 22 (2012) 2387-2391.

[134] C. Fang, N.M. Bandaru, A.V. Ellis, N.H. Voelcker, Beta-cyclodextrin decorated nanostructured SERS substrates facilitate selective detection of endocrine disruptor chemicals, Biosens. Bioelectron. 42 (2013) 632-639.

[135] L.L. He, M.S. Lin, H. Li, N.J. Kim, Surface-enhanced Raman spectroscopy coupled with dendritic silver nanosubstrate for detection of restricted antibiotics, J. Raman Spectrom. 41 (2010) 739-744.

[136] L.G. Hu, S.Y. Xu, C.S. Pan, C.G. Yuan, H.F. Zou, G.B. Jiang, Matrix-assisted laser desorption/ionization time-of-flight mass spectrometry with a matrix of carbon nanotubes for the analysis of low-mass compounds in environmental samples, Environ. Sci. Technol. 39 (2005) 8442-8447.

[137] J.Y. Liu, Y. Liu, M.X. Gao, X.M. Zhang, High throughput detection of tetracycline residues in milk using graphene or graphene oxide as MALDITOF MS matrix, J. Am. Soc. Mass Spectrom. 23 (2012) 1424-1427.

[138] H. Kawasaki, K. Nakai, R. Arakawa, E.K. Athanassiou, R.N. Grass, W.J. Stark, Functionalized graphene-coated cobalt nanoparticles for highly efficient surface-assisted laser desorption/ionization mass spectrometry analysis, Anal. Chem. 84 (2012) 9268-9275. 
[139] Q. Liu, M.T. Cheng, G.B. Jiang, Mildly oxidized graphene: Facile synthesis, characterization, and application as a matrix in MALDI mass spectrometry, Chem. Eur. J. 19 (2013) 5561-5565.

[140] C. Wang, S. de Rooy, C.F. Lu, V. Fernand, L. Moore, P. Berton, I.M. Warner, An immobilized graphene oxide stationary phase for open-tubular capillary electrochromatography, Electrophoresis 34 (2013) 1197-1202.

[141] H.B. Noh, K.S. Lee, B.S. Lim, S.J. Kim, Y.B. Shim, Total analysis of endocrine disruptors in a microchip with gold nanoparticles, Electrophoresis 31 (2010) 3053-3060.

[142] M. Frasconi, R. Tel-Vered, M. Riskin, I. Willner, Surface plasmon resonance analysis of antibiotics using imprinted boronic acid-functionalized $\mathrm{Au}$ nanoparticle composites, Anal. Chem. 82 (2010) 2512-2519.

[143] F. Fernandez, F. Sanchez-Baeza, M.P. Marco, Nanogold probe enhanced surface plasmon resonance immunosensor for improved detection of antibiotic residues, Biosens. Bioelectron. 34 (2012) 151-158.
[144] P. Zhao, G. Cao, L. Zhou, Q. Liu, M. Guo, Y. Huang, Q. Cai, S. Yao, Nitrate enhanced electrochemiluminescence determination of tris(2,3dibromopropyl) isocyanurate with a gold nanoparticles-modified gold electrode, Analyst 136 (2011) 1952-1956.

[145] Z.L. Jiang Y. Li, A.H. Liang A.M. Oin, A sensitive and selective immunonanogold resonance-scattering spectral method for the determination of trace penicillin G, Luminescence 23 (2008) 157-162.

[146] D.M. Yao, G.Q. Wen, Z.L. Jiang, A highly sensitive and selective resonance Rayleigh scattering method for bisphenol A detection based on the aptamernanogold catalysis of the $\mathrm{HAuCl}_{4}$-vitamin C particle reaction, RSC Adv. 3 (2013) 13353-13356.

[147] J.W. Park, S. Kurosawa, H. Aizawa, Y. Goda, M. Takai, K. Ishihara, Piezoelectric immunosensor for bisphenol A based on signal enhancing step with 2 methacrolyloxyethyl phosphorylcholine polymeric nanoparticle, Analyst 131 (2006) 155-162. 